\title{
Fault Detection in Uncertain LPV Systems with Imperfect Scheduling Parameter Using Sliding Mode Observers
}

\author{
Kumar Pakki Bharani Chandra, Halim Alwi and Christopher Edwards
}

\begin{abstract}
This paper presents a sliding mode fault detection scheme for linear parameter varying (LPV) systems with uncertain or imperfectly measured scheduling parameters. In the majority of LPV systems, it is assumed that the scheduling parameters are exactly known. In reality due to noise or possibly faulty sensors, it is sometimes impossible to have accurate knowledge of the scheduling parameters and a design based on the assumption of perfect knowledge of the scheduling parameters cannot be guaranteed to work well in this situation. This paper proposes a sliding mode observer scheme to reconstruct actuator and sensor faults in a situation where the scheduling parameters are imperfectly known. The efficacy of the approach is demonstrated on simulation data taken from the nonlinear RECONFIGURE benchmark model
\end{abstract}

Keywords: Fault detection, sliding modes, observers

\section{INTRODUCTION}

Fault detection and isolation (FDI) plays an important role in many engineering applications - especially in the aerospace, electrical machinery and process control industries. An effective fault detection scheme, when used with appropriate controllers can maintain the integrity of the closed-loop system in the presence of faults. One popular approach for designing FDI schemes is based on observer concepts, usually based on linear theory or slightly extended versions. A wide range of observer paradigms have been considered in the literature: for example Kalman filters and their extensions (see for example [1], [2], [3]), $\mathcal{H}_{\infty}$ filters (see for example [4], [5], [6]), unknown input observers (see for example [7], [8], [9]), and sliding mode observers (SMOs) (see for example [10], [11], [12]). One approach to extending linear methods to make them work effectively across the whole plant operating range is to design (linear) observers at select operating points, and then schedule the gains with respect to certain parameters [4]. One of the main difficulties with gain scheduling is the selection of the operating points and how to choose the gains at intermediate points. Furthermore, formally, between the operating points the stability of the observer cannot be guaranteed.

A more rigorous alternative approach is based on so-called linear parameter varying (LPV) system designs. In the LPV approach the gains are automatically scheduled with respect to the plant varying parameters. Another advantage is many nonlinear systems can be naturally approximated by LPV systems [13], [14]. Recent results on LPV based fault detection schemes appears in [15], [16], [17] from work in the EU-funded ADDSAFE project. Recently several sliding mode observer structures have been proposed for LPV systems (see for example [18], [19], [20]). However in all these papers the scheduling parameters are assumed to be perfectly known. However in reality this may not be the case: for example inaccurate sensors and/or faults can lead to imperfect knowledge of the parameters. The use of this corrupted information will affect the performance of the observer. The design of Luenberger-like observers in scenarios involving uncertain scheduling parameters has been investigated. In [21], using a Linear matrix inequality (LMI) framework an LPV observer was proposed. Later, a controller and a combined controllerobserver scheme for inexact continuous LPV polytopic systems was presented in [22], [23]. In [24], [25] $\mathcal{H}_{\infty}$ filters were employed to deal with uncertainty in the scheduling parameters; exploiting parameter dependent LMI methods. More recently, an $\mathcal{H}_{\infty}$ filter has been proposed to deal with both additive and multiplicative uncertainties in the LPV parameters in [26]. Recent applications of these ideas to aerospace systems have been explored in [27], [28]. A fault reconstruction scheme for LPV systems with perfect scheduling parameter knowledge, using $\mathcal{H}_{\infty}$ methods has been investigated in [29]. Very few papers have addressed specifically the fault reconstruction problem for LPV systems with uncertain scheduling parameters, notable exceptions are [30]. To the authors' knowledge,

K.P.B. Chandra is with GMR Institute of Technology, Rajam - 532127, AP, India. bharanichandrakumar.pegmrit.org

H. Alwi and C. Edwards are with the College of Engineering, Mathematics and Physical Sciences, University of Exeter, EX4 4QF, UK. h.alwi@exeter.ac.uk, C.Edwards@exeter.ac.uk 
fault reconstruction for LPV systems with uncertain scheduling parameters, using sliding mode observers, has not yet been investigated.

This paper presents a new FDI scheme based on sliding mode ideas for LPV systems with uncertain scheduling parameters. This exploits the fact that sliding mode observers have an inherent ability for fault reconstruction, due to the unique property of the equivalent injection signal [11]. This work is a direct extension of [20], where it was assumed that the scheduling parameters are perfectly measurable. An earlier version of this work was presented in [31] and dealt with an actuator fault problem. In [31] all the simulations were performed on simplified LPV models, whereas in this paper, the work has been extended to deal with sensor faults and the simulation results are based on data from a high-fidelity nonlinear aircraft model [32]. The main contributions of this paper are: (a)

1) the systematic development of a SMO for a class of LPV systems to reconstruct faults;

2) the proposed SMO is specifically developed to handle uncertain scheduling parameters;

3) the proposed approach is validated on data from the RECONFIGURE benchmark [32] which represents a high-fidelity nonlinear aircraft model.

\section{LineAR PARAMETER VARYing SyStem}

Consider an uncertain LPV system subject to actuator faults written in the form

$$
\begin{aligned}
\dot{x}(t) & =A(\rho) x(t)+B(\rho) u(t)+H(\rho) f_{i}(t)+M \xi(t, y, u) \\
y(t) & =C x(t)+d(t),
\end{aligned}
$$

where the parameter varying matrices $A(\rho) \in \mathbb{R}^{n \times n}, B(\rho) \in \mathbb{R}^{n \times m}$ and $H(\rho) \in \mathbb{R}^{n \times q}$. The fixed matrices are $M \in \mathbb{R}^{n \times k}$ and $C \in \mathbb{R}^{p \times n}$. In (1) and (2) the state vector is $x \in \mathbb{R}^{n}, y \in \mathbb{R}^{p}$ is the measured output vector and $u \in \mathbb{R}^{m}$ is the control signal. The vector $\rho \in \Omega \subset \mathbb{R}^{r}$ denotes the varying scheduling parameters, $f_{i}(t) \in \mathbb{R}^{q}$ models the unknown actuator faults, $\xi(t, y, u) \in \mathbb{R}^{k}$ captures the uncertainty in the system and the signal $d(t)$ represents an ever-present corruption of the true measured outputs but does not represent sensor noise. As in [20], three assumptions will be made:

Assumption 1. The signal $d(t)$ is of low frequency and its derivative satisfies

$$
\dot{d}(t)=-a_{f} d(t)+a_{f} \phi(t),
$$

where $a_{f}$ is a positive scalar and $\phi(t)$ is an unknown input signal. In the frequency domain, the $d(t)$ will have low frequency characteristics. Note that $d(t)$ represents corruption in the measurements which are always present even in the absence of sensor faults, and in general this is accounted in the controller design. In some situations, such as one of the scenarios from the ADDSAFE benchmark problem [17], the unknown sensor faults are also of low frequency and hence the lower frequency components of $d(t)$ can cause false alarms. Hence solely for design purposes, $d(t)$ is assumed as a low frequency signal [17]. Note that the signal $d(t)$ does not represent sensor noise - during the SMO design sensor noise is not explicitly considered.

Assumption 2. The matrix $H(\rho)$ can be factorised as

$$
H(\rho)=F E(\rho)
$$

where $F \in \mathbb{R}^{n \times q}$ is fixed and the square matrix $E(\rho) \in \mathbb{R}^{q \times q}$ is parameter varying. Furthermore it is assumed that $\operatorname{det}(E(\rho)) \neq 0$ for all $\rho \in \Omega$.

Assumption 3. The matrix $C$ has full rank and $\operatorname{rank}(C F)=q$.

As a consequence of Assumption 3, there exists a coordinate transformation $x(t) \mapsto T_{o} x(t)$ such that in the new coordinates

$$
y(t)=\left[\begin{array}{ll}
0 & T
\end{array}\right]\left[\begin{array}{l}
x_{1}(t) \\
x_{2}(t)
\end{array}\right]+d(t),
$$

where $T \in \mathbb{R}^{p \times p}$ is orthogonal, and

$$
F=\left[\begin{array}{c}
0_{(n-p) \times q} \\
F_{2}
\end{array}\right]=\left[\begin{array}{c}
0_{(n-p) \times q} \\
0 \\
F_{0}
\end{array}\right],
$$

where $F_{2} \in \mathbb{R}^{p \times q}$ and $F_{0} \in \mathbb{R}^{q \times q}$. For details see [20]. 
Using Assumptions 1-3 and (1) and (4), the system can be written as

$$
\begin{aligned}
\dot{x}(t) & =A(\rho) x(t)+B(\rho) u(t)+F E(\rho) f_{i}(t)+M \xi(t, y, u) \\
& =A(\rho) x(t)+B(\rho) u(t)+F f_{v}(t, \rho)+M \xi(t, y, u),
\end{aligned}
$$

where the 'virtual faults' $f_{v}(t):=E(\rho) f_{i}(t)$. In what follows, an observer will be first designed to estimate the virtual faults $f_{v}(t, \rho)$ and then the actual fault $f_{i}(t)$ will be recovered by exploiting the fact that $E(\rho)$ is invertible.

\section{Sliding Mode Observer for Uncertain Linear Parameter Varying System}

\section{A. Structure of the Observer}

Consider a (sliding mode) observer of the form given by

$$
\begin{aligned}
\dot{\hat{x}}(t) & =A(\hat{\rho}) \hat{x}(t)+B(\hat{\rho}) u(t)-G_{l}(\hat{\rho}) e_{y}(t)+G_{n} \nu(t) \\
\hat{y}(t) & =C \hat{x}(t),
\end{aligned}
$$

where $\hat{\rho}$ is the set of (estimated) observer scheduling parameters. In (8) and (9) the matrices $G_{l}(\hat{\rho}), G_{n} \in \mathbb{R}^{n \times p}$ are the observer gains and represent design freedom. In (8), $\nu(t)$ is a discontinuous nonlinear function which will be explicitly defined later in the paper used to induce a sliding motion. In this paper it will be assumed that typically $\rho(t) \neq \hat{\rho}(t)$ and an observer will be presented to estimate the faults despite imperfect knowledge of the scheduling parameters. The estimate of the fault $f_{i}(t)$ will be obtained by scaling the so-called equivalent injection signal [11] (whilst sliding). Specifically

$$
\hat{f}_{i}=E^{-1}(\rho) W T^{\mathrm{T}} \nu_{e q}
$$

where $W \in \mathbb{R}^{q \times p}$ is design freedom and $\nu_{e q}$ represent the equivalent injection (the 'average' value of $\nu(t)$ ) required to maintain sliding [11].

Define the state estimation error as $e(t)=\hat{x}(t)-x(t)$ and partition the error conformably with the partitions in (5) and (6) so that $e(t)=\operatorname{col}\left(e_{1}(t), e_{2}(t)\right)$. The error between the estimated and actual output is given by

$$
\begin{aligned}
e_{y}(t) & =\hat{y}(t)-y(t)=C e(t)-d(t) \\
& =\left[\begin{array}{ll}
0 & T
\end{array}\right]\left[\begin{array}{l}
e_{1}(t) \\
e_{2}(t)
\end{array}\right]-d(t)=T e_{2}(t)-d(t) .
\end{aligned}
$$

The objective is to force the output estimation error $e_{y}(t)$ to zero in finite time and induce a sliding motion on the surface

$$
\mathcal{S}=\left\{e(t) \in \mathbb{R}^{n}, e_{y}(t)=0\right\}
$$

Subtracting (7) from (8), the state estimation error satisfies the differential equation

$$
\dot{e}(t)=A(\hat{\rho}) e+(A(\hat{\rho})-A(\rho)) x(t)+(B(\hat{\rho})-B(\rho)) u(t)-G_{l}(\hat{\rho}) e_{y}(t)+G_{n} \nu(t)-F f_{v}(t)-M \xi(t, y, u) .
$$

The structure of $G_{n}$ associated with the nonlinear injection term is assumed, as in [20], to have the form

$$
G_{n}=\left[\begin{array}{l}
G_{n 1} \\
G_{n 2}
\end{array}\right]=\left[\begin{array}{c}
-L T^{\mathrm{T}} \\
T^{\mathrm{T}}
\end{array}\right]
$$

where the matrix sub-block $L \in \mathbb{R}^{(n-p) \times p}$ is in turn assumed to be of the form

$$
L=\left[\begin{array}{ll}
L_{1} & 0
\end{array}\right] \text {, }
$$

where $L_{1} \in \mathbb{R}^{(n-p) \times(p-q)}$. In the analysis which follows (13) will be written in partitioned form as

$$
\begin{aligned}
& {\left[\begin{array}{c}
\dot{e}_{1}(t) \\
\dot{e}_{2}(t)
\end{array}\right]=\left[\begin{array}{ll}
A_{11}(\hat{\rho}) & A_{12}(\hat{\rho}) \\
A_{21}(\hat{\rho}) & A_{22}(\hat{\rho})
\end{array}\right]\left[\begin{array}{l}
e_{1}(t) \\
e_{2}(t)
\end{array}\right]+\left[\begin{array}{c}
B_{1}^{e}(\hat{\rho}, \rho) \\
B_{2}^{e}(\hat{\rho}, \rho)
\end{array}\right] u(t)+\left[\begin{array}{cc}
A_{11}^{e}(\hat{\rho}, \rho) & A_{12}^{e}(\hat{\rho}, \rho) \\
A_{21}^{e}(\hat{\rho}, \rho) & A_{22}^{e}(\hat{\rho}, \rho)
\end{array}\right]\left[\begin{array}{l}
x_{1}(t) \\
x_{2}(t)
\end{array}\right]} \\
& -\left[\begin{array}{c}
0 \\
F_{2}
\end{array}\right] f_{v}(t)+\left[\begin{array}{l}
G_{n 1} \\
G_{n 2}
\end{array}\right] \nu(t)-\left[\begin{array}{l}
G_{l 1}(\hat{\rho}) \\
G_{l 2}(\hat{\rho})
\end{array}\right] e_{y}(t)-\left[\begin{array}{c}
M_{1} \\
M_{2}
\end{array}\right] \xi(t, y, u),
\end{aligned}
$$

where $A_{i, j}(\hat{\rho})$ and $A_{i, j}(\rho)$ for $i, j \in\{1,2\}$ represent appropriate partitions of $A(\hat{\rho})$ and $A(\rho)$, and $B_{i}(\hat{\rho})$ and $B_{i}(\rho)$ are sub-blocks from $B(\hat{\rho})$ and $B(\rho)$. Further $A_{i j}^{e}(\hat{\rho}, \rho):=A_{i j}(\hat{\rho})-A_{i j}(\rho)$ and $B_{i}^{e}(\hat{\rho}, \rho):=B_{i}(\hat{\rho})-B_{i}(\rho)$ for $i, j \in\{1,2\}$. 
For analysis purposes, define a linear coordinate transformation $\left(e_{1}, e_{2}\right) \mapsto\left(e_{1}+L e_{2}, T e_{2}\right):=\left(\tilde{e}_{1}, \tilde{e}_{2}\right)$, where

$$
T_{L}=\left[\begin{array}{ll}
I & L \\
0 & T
\end{array}\right]
$$

In the new set of coordinates, equation (16) becomes

$$
\begin{aligned}
& {\left[\begin{array}{c}
\dot{\tilde{e}}_{1}(t) \\
\dot{\tilde{e}}_{2}(t)
\end{array}\right]=\underbrace{\left[\begin{array}{cc}
\tilde{A}_{11}(\hat{\rho}) & \tilde{A}_{12}(\hat{\rho}) \\
\tilde{A}_{21}(\hat{\rho}) & \tilde{A}_{22}(\hat{\rho})
\end{array}\right]}_{\tilde{A}(\hat{\rho})=T_{L} A(\hat{\rho}) T_{L}^{-1}}\left[\begin{array}{c}
\tilde{e}_{1}(t) \\
\tilde{e}_{2}(t)
\end{array}\right]+\underbrace{\left[\begin{array}{c}
\tilde{B}_{1}^{e}(\hat{\rho}, \rho) \\
\tilde{B}_{2}^{e}(\hat{\rho}, \rho)
\end{array}\right]}_{\tilde{B}^{e}(\hat{\rho}, \rho)=T_{L} B^{e}(\hat{\rho}, \rho)} u(t)+\underbrace{\left[\begin{array}{cc}
\tilde{A}_{11}^{e}(\hat{\rho}, \rho) & \tilde{A}_{12}^{e}(\hat{\rho}, \rho) \\
\tilde{A}_{21}^{e}(\hat{\rho}, \rho) & \tilde{A}_{22}^{e}(\hat{\rho}, \rho)
\end{array}\right]}_{\tilde{A}^{e}(\hat{\rho}, \rho)=T_{L} A^{e}(\hat{\rho}, \rho) T_{L}^{-1}}\left[\begin{array}{c}
x_{1}(t) \\
x_{2}(t)
\end{array}\right]} \\
& -\underbrace{\left[\begin{array}{c}
0 \\
\tilde{F}_{2}
\end{array}\right]}_{\tilde{F}=T_{L} F} f_{v}(t)+\underbrace{\left[\begin{array}{c}
0 \\
I
\end{array}\right]}_{\tilde{G}_{n}=T_{L} G_{n}} \nu(t)-\underbrace{\left[\begin{array}{c}
\tilde{G}_{l 1}(\hat{\rho}) \\
\tilde{G}_{l 2}(\hat{\rho})
\end{array}\right]}_{\tilde{G}_{l}=T_{L} G_{l}} e_{y}(t)-\underbrace{\left[\begin{array}{c}
\tilde{M}_{1} \\
\tilde{M}_{2}
\end{array}\right]}_{\tilde{M}=T_{L} M} \xi(t, y, u) .
\end{aligned}
$$

Furthermore, in (17), it can be shown $\tilde{A}_{11}(\hat{\rho})=A_{11}(\hat{\rho})+L A_{21}(\hat{\rho})$. In the new coordinates $\tilde{C}=C T_{L}^{-1}=\left[\begin{array}{ll}0 & I\end{array}\right]$, and consequently the output error can be expressed as

$$
e_{y}=\tilde{e}_{2}-d
$$

Due to the special structure of $L$ in (15), if $A_{21}(\hat{\rho})$ from (16) is further partitioned as

$$
A_{21}(\hat{\rho})=\left[\begin{array}{l}
A_{211}(\hat{\rho}) \\
A_{212}(\hat{\rho})
\end{array}\right]
$$

then $\tilde{A}_{11}(\hat{\rho})$ can be written as $\tilde{A}_{11}(\hat{\rho})=A_{11}(\hat{\rho})+L_{1} A_{211}(\hat{\rho})$.

Assumption 4. There exists a symmetric positive definite matrix, $P_{11}$, such that

$$
\tilde{A}_{11}^{T}(\hat{\rho}) P_{11}+P_{11} \tilde{A}_{11}(\hat{\rho})<0 .
$$

for all $\hat{\rho} \in \Omega$; i.e. in other words $\tilde{A}_{11}(\hat{\rho})$ is quadratically stable.

Finally create a new state vector by augmenting $d(t)$ from (3), and $\tilde{e}_{1}(t)$ and $e_{y}(t)$ from (17) and (18), such that

$$
\begin{aligned}
& {\left[\begin{array}{c}
\dot{d}(t) \\
\dot{\tilde{e}}_{1}(t) \\
\hline \dot{e}_{y}(t)
\end{array}\right]=\underbrace{\left[\begin{array}{cc|c}
-a_{f} I_{p} & 0 & 0 \\
\tilde{A}_{12}(\hat{\rho}) & \tilde{A}_{11}(\hat{\rho}) & \tilde{A}_{12}(\hat{\rho}) \\
\hline \tilde{A}_{22}(\hat{\rho})+a_{f} & \tilde{A}_{21}(\hat{\rho}) & \tilde{A}_{22}(\hat{\rho})
\end{array}\right]}_{\tilde{A}_{a}(\hat{\rho})}\left[\begin{array}{c}
d(t) \\
\tilde{e}_{1}(t) \\
\hline e_{y}(t)
\end{array}\right]+\underbrace{\left[\begin{array}{cccc}
0 & 0 & 0 & a_{f} T \\
\tilde{A}_{1}^{e}(\rho, \hat{\rho}) & \tilde{B}_{1}^{e}(\rho, \hat{\rho}) & -\tilde{M}_{1} & 0 \\
\hline \tilde{A}_{2}^{e}(\rho, \hat{\rho}) & \tilde{B}_{2}{ }^{e}(\rho, \hat{\rho}) & -\tilde{M}_{2} & -a_{f} T
\end{array}\right]}_{\tilde{M}_{a}(\rho, \hat{\rho})} \xi_{a}(t, y, u)} \\
& -\left[\begin{array}{c}
0 \\
0 \\
\hline \tilde{F}_{2}
\end{array}\right] f_{v}(t)-\left[\begin{array}{c}
0 \\
\tilde{G}_{l 1}(\hat{\rho}) \\
\hline \tilde{G}_{l 2}(\hat{\rho})
\end{array}\right] e_{y}(t)+\left[\begin{array}{c}
0 \\
0 \\
\hline I
\end{array}\right] \nu(t)
\end{aligned}
$$

where

$$
\begin{aligned}
\tilde{A}_{1}^{e}(\hat{\rho}, \rho) & =\left[\begin{array}{ll}
\tilde{A}_{11}^{e}(\hat{\rho}, \rho) & \tilde{A}_{12}^{e}(\hat{\rho}, \rho)
\end{array}\right] \\
\tilde{A}_{2}^{e}(\hat{\rho}, \rho) & =\left[\begin{array}{ll}
\tilde{A}_{21}^{e}(\hat{\rho}, \rho) & \tilde{A}_{22}^{e}(\hat{\rho}, \rho)
\end{array}\right] .
\end{aligned}
$$

and

$$
\xi_{a}(t, y, u)=\operatorname{col}\left(x(t), u(t), \xi(t, y, u), T^{\mathrm{T}} \phi(t)\right) .
$$

Define

$$
\left[\begin{array}{c|c}
\tilde{A}_{a 11}(\hat{\rho}) & \tilde{A}_{a 12}(\hat{\rho}) \\
\hline \tilde{A}_{a 21}(\hat{\rho}) & \tilde{A}_{a 22}(\hat{\rho})
\end{array}\right]:=\left[\begin{array}{cc|c}
-a_{f} I_{p} & 0 & 0 \\
\tilde{A}_{12}(\hat{\rho}) & \tilde{A}_{11}(\hat{\rho}) & \tilde{A}_{12}(\hat{\rho}) \\
\hline \tilde{A}_{22}(\hat{\rho})+a_{f} & \tilde{A}_{21}(\hat{\rho}) & \tilde{A}_{22}(\hat{\rho})
\end{array}\right],
$$

and

$$
\left[\begin{array}{c}
\tilde{M}_{a 1}(\hat{\rho}, \rho) \\
\tilde{M}_{a 2}(\hat{\rho}, \rho)
\end{array}\right]:=\left[\begin{array}{cccc}
0 & 0 & 0 & a_{f} T \\
\tilde{A}_{1}^{e}(\hat{\rho}, \rho) & \tilde{B}_{1}^{e}(\hat{\rho}, \rho) & -\tilde{M}_{1} & 0 \\
\hline \tilde{A}_{2}^{e}(\hat{\rho}, \rho) & \tilde{B}_{2}^{e}(\hat{\rho}, \rho) & -\tilde{M}_{2} & -a_{f} T
\end{array}\right]
$$


then one choice for the observer gain $\tilde{G}_{l}(\hat{\rho})$ (in the new coordinates) is

$$
\tilde{G}_{l}(\hat{\rho}):=\left[\begin{array}{c}
\tilde{G}_{l 1}(\hat{\rho}) \\
\tilde{G}_{l 2}(\hat{\rho})
\end{array}\right]:=\left[\begin{array}{c}
\tilde{A}_{12}(\hat{\rho}) \\
\tilde{A}_{22}(\hat{\rho})-\tilde{A}_{22}^{s}
\end{array}\right]
$$

where $\tilde{A}_{22}^{s}$ is any selected Hurwitz matrix. Substituting $\tilde{G}_{l}(\hat{\rho})$ from (25) into (21) yields

$$
\underbrace{\left[\begin{array}{c}
\dot{e}_{a 1}(t) \\
\dot{e}_{y}(t)
\end{array}\right]}_{\tilde{\tilde{e}}_{a}}=\underbrace{\left[\begin{array}{cc}
\tilde{A}_{a 11}(\hat{\rho}) & 0 \\
\tilde{A}_{a 21}(\hat{\rho}) & \tilde{A}_{22}^{s}
\end{array}\right]}_{\tilde{A}_{o}(\hat{\rho})} \underbrace{\left[\begin{array}{c}
e_{a 1}(t) \\
e_{y}(t)
\end{array}\right]}_{\tilde{e}_{a}}+\underbrace{\left[\begin{array}{c}
\tilde{M}_{a 1}(\hat{\rho}, \rho) \\
\tilde{M}_{a 2}(\hat{\rho}, \rho)
\end{array}\right]}_{\tilde{M}_{a}(\hat{\rho}, \rho)} \xi_{a}(t, y, u)-\underbrace{\left[\begin{array}{c}
0 \\
\tilde{F}_{2}
\end{array}\right]}_{\tilde{F}_{a}} f_{v}(t)+\underbrace{\left[\begin{array}{c}
0 \\
I
\end{array}\right]}_{\tilde{G}_{n_{a}}} \nu(t) .
$$

Here the discontinuous output error injection term is defined as

$$
\nu(t)=-\lambda(t, y, u, \hat{\rho}) \frac{P_{o} e_{y}(t)}{\left\|P_{o} e_{y}(t)\right\|},
$$

where $P_{o}$ is a symmetric positive definite Lyapunov matrix for the Hurwitz matrix $\tilde{A}_{22}^{s}$. The modulation gain $\lambda(\cdot)$ is a scalar satisfying $\lambda(\cdot)>\left\|\tilde{F}_{2}\right\| a(t, \hat{\rho}, u)+\eta_{0}$, where the function $a(t, \hat{\rho}, u)$ is a known bound on the fault such that $\left\|f_{v}(t, \hat{\rho})\right\| \leq a(t, \hat{\rho}, u)$, and $\eta_{0}$ is a positive scalar.

Remark 1: From (14), (25) and (27), the design freedom in the observer configuration in (8)-(9) is characterized in terms of a gain matrix $L_{1}$ from (15) (subject to satisfying Assumption 4), and the Hurwitz matrix $\tilde{A}_{22}^{s}$ in (25).

Lemma 1. [33] If there exists a symmetric positive definite matrix (s.p.d) $P_{11}$ such that (20) holds, then there exists a s.p.d $\tilde{P}_{a}$ s.t. $\tilde{P}_{a} \tilde{A}_{o}(\hat{\rho})+\tilde{A}_{o}^{T}(\hat{\rho}) \tilde{P}_{a}<-\tilde{Q}_{a}$ where $\tilde{Q}_{a}$ is s.p.d and $\tilde{A}_{o}(\hat{\rho})$ is defined in (26).

Proof. The proof is similar to the one given in [33].

Let $b$ and $b_{\phi}$ be scalars representing upper bounds on the uncertainty and measurement perturbations so that conditions

$$
\|\xi(t, y, u)\|<b \quad \text { and } \quad\|\phi(t)\|<b_{\phi}
$$

hold. Also assume that, despite imperfect information about $\rho$, the closed-loop system satisfies

$$
\|x(t)\|<b_{x} \quad \text { and } \quad\|u(t)\|<b_{u}
$$

so that the aggregated disturbance defined in (22) satisfies $\left\|\xi_{a}(t, y, u)\right\|<\bar{b}$, where $\bar{b}$ is a positive scalar. Define a scalar $\mu_{0}=\lambda_{\min }\left(\tilde{Q}_{a}\right)>0$. Then it follows from Lemma 1 that

$$
\tilde{P}_{a} \tilde{A}_{o}(\hat{\rho})+\tilde{A}_{o}^{\mathrm{T}}(\hat{\rho}) \tilde{P}_{a} \leq-\mu_{0} I .
$$

Lemma 2. [33] The state estimation error $\tilde{e}_{a}$ in (26) is ultimately bounded with respect to the compact set defined

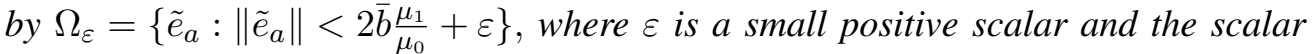

$$
\mu_{1}=\sqrt{\lambda_{\max }\left(\tilde{M}_{a}(\hat{\rho}, \rho)^{T} \tilde{P}_{a}^{2} \tilde{M}_{a}(\hat{\rho}, \rho)\right)},
$$

where $\tilde{M}_{a}$ is defined in (21).

Proof. The proof is similar to the one given in [33].

Based on the scalars from Lemma 1 and 2, a suitable choice of the modulation gain in (27) will be identified.

Lemma 3. If the modulation gain in (27) satisfies

$$
\lambda(\cdot) \geq 2 a_{21} \bar{b} \frac{\mu_{1}}{\mu_{0}}+\left\|\tilde{M}_{a_{2}}\right\| \bar{b}+\left\|\tilde{F}_{2}\right\| a+\eta_{0}
$$

where the scalar $a_{21}=\max _{\hat{\rho} \in \Omega} \tilde{A}_{a_{21}}(\hat{\rho})$ then an ideal sliding motion takes place on $\mathcal{S}$ given in (12).

Proof. Consider a Lyapunov function candidate $V=e_{y}^{\mathrm{T}} P_{o} e_{y}$, where $P_{o}$ is a s.p.d Lyapunov matrix for $\tilde{A}_{22}^{s}$. The derivative of $V$ along the trajectory of $e_{y}$ in (26) is

$$
\begin{aligned}
\dot{V} & =2 e_{y}^{\mathrm{T}} P_{o} \tilde{A}_{a_{21}}(\hat{\rho}) \tilde{e}_{a_{1}}-e_{y}^{\mathrm{T}}\left(P_{o} \tilde{A}_{22}^{s}+\left(\tilde{A}_{22}^{s}\right)^{\mathrm{T}} P_{o}\right) e_{y}+2 e_{y}^{\mathrm{T}} P_{o} \nu(t)+2 e_{y}^{\mathrm{T}} P_{o} \tilde{M}_{a_{2}}(\hat{\rho}, \rho) \xi_{a}(t, y, u)-2 e_{y}^{\mathrm{T}} P_{o} \tilde{F}_{2} f_{\nu}(t) \\
& \leq-2\left\|P_{o} e_{y}\right\|\left(\lambda-a_{21}\left\|\tilde{e}_{a_{1}}\right\|-\left\|\tilde{F}_{2}\right\|\left\|f_{\nu}\right\|-\left\|\tilde{M}_{a_{2}}(\hat{\rho}, \rho)\right\|\left\|\xi_{a}(t, y, u)\right\|\right),
\end{aligned}
$$


since $e_{y}^{\mathrm{T}}\left(P_{o} \tilde{A}_{22}+\left(\tilde{A}_{22}\right)^{\mathrm{T}} P_{o}\right) e_{y} \leq 0$ by choice of $P_{o}$.

From Lemma 2, the estimation error $\tilde{e}_{a_{1}}$ is bounded by $\left\|\tilde{e}_{a_{1}}\right\|<2 \bar{b}_{\frac{\mu_{1}}{\mu_{0}}}+\varepsilon$, and specifically $\tilde{e}_{a} \in \Omega_{\varepsilon}$ in finite time. Using the fact that $\left\|f_{\nu}\right\|<a(t, \rho, u)$, and $\left\|\xi_{a}(t, y, u)\right\|<\bar{b}$, and from the choice of $\lambda$ in (29), (30) can be written as $\dot{V} \leq-2 \eta_{0}\left\|P_{o} e_{y}\right\| \leq-2 \eta_{0} \eta \sqrt{V}$ where $\eta:=\sqrt{\lambda_{\min }\left(P_{o}\right)}$. This ensures the output estimation error $e_{y}$ reaches zero in finite time and sliding takes place on $\mathcal{S}$ in finite time.

Define the reconstruction signal for the unknown input $f_{v}(t)$ (the virtual fault) to be

$$
\hat{f}_{v}(t)=W T^{\mathrm{T}} \nu_{e q}(t)
$$

where $W \in \mathbb{R}^{q \times p}$ has the form $W=\left[\begin{array}{ll}W_{1} & F_{0}^{-1}\end{array}\right]$, and $W_{1} \in \mathbb{R}^{q \times(p-q)}$ represents design freedom. The square matrix and $F_{0}$ is defined in (6) as part of the fault input distribution matrix.

It follows from (26) that during sliding motion

$$
\dot{e}_{a}(t)=A_{a}(\hat{\rho}) e_{a}(t)+B_{a}(\hat{\rho}, \rho) \xi_{a}(t, y, u)
$$

where

$$
e_{a}(t)=\operatorname{col}\left(T d(t), \tilde{e}_{1}(t)\right)
$$

and the system matrices in (32) are given by

$$
\begin{aligned}
A_{a} & =\left[\begin{array}{cc}
-a_{f} I_{p} & 0 \\
A_{12}(\hat{\rho})+L A_{22}(\hat{\rho})+a_{f} L & A_{11}(\hat{\rho})+L A_{21}(\hat{\rho})
\end{array}\right] \\
B_{a} & =\left[\begin{array}{cccc}
0 & 0 & 0 & a_{f} I_{p} \\
A_{1}^{e}(\hat{\rho}, \rho)+L A_{2}^{e}(\hat{\rho}, \rho) & B_{1}^{e}(\hat{\rho}, \rho)+L B_{2}^{e}(\hat{\rho}, \rho) & -\left(M_{1}+L M_{2}\right) & -a_{f} L
\end{array}\right] .
\end{aligned}
$$

Note that for all $W_{1}, W F_{2}=I_{q}$. It follows from (31) and (26) that the fault estimation error is

$$
\hat{f}_{v}(t)-f_{v}(t)=C_{a}(\hat{\rho}) e_{a}(t)+D_{a}(\hat{\rho}, \rho) \xi_{a}(t, y, u),
$$

where

$$
\begin{aligned}
C_{a} & =\left[\begin{array}{lll}
-W A_{22}(\hat{\rho})-a_{f} W & -W A_{21}(\hat{\rho})
\end{array}\right] \\
D_{a} & =\left[\begin{array}{llll}
-W A_{2}^{e}(\hat{\rho}, \rho) & -W B_{2}^{e}(\hat{\rho}, \rho) & W M_{2} & W a_{f}
\end{array}\right] .
\end{aligned}
$$

Equations (32) and (36) constitute a state space representation where $e_{a}(t)$ and $\hat{f}_{v}(t)-f_{v}(t)$ can be considered as the state and output vectors, $A_{a}(\hat{\rho}), B_{a}(\hat{\rho}, \rho), C_{a}(\hat{\rho})$ and $D_{a}(\hat{\rho}, \rho)$ as state space matrices, and the system is driven by the disturbance $\xi_{a}(t, y, u)$. In this paper the objective is to minimise the effect of $\xi_{a}(\cdot)$ on the fault reconstruction error $\hat{f}_{v}(t)-f_{v}(t)$ in an $\mathcal{L}_{2}$ sense. The key point is that minimising the effect of $\xi_{a}$ on $\hat{f}_{v}(t)-f_{v}(t)$ also minimises the effect of the observer plant mismatch when $\rho \neq \hat{\rho}$.

Theorem 1. Consider the observer in (8)-(9) with gains parameterized in terms of $L$ from (15) and the nonlinear modulation gain $\lambda(t, y, u, \hat{\rho})$ from (27) chosen to ensure Lemma 3 is satisfied. Then if by choice of L, a positive definite matrix $P$ can be found so that

$$
\begin{aligned}
& {\left[\begin{array}{ccc}
A_{a}^{T}(\hat{\rho}) P+P A_{a}(\hat{\rho}) & P\left(B_{a}(\hat{\rho}, \rho) \Delta\right) & C_{a}^{T}(\hat{\rho}) \\
\left(B_{a}(\hat{\rho}, \rho) \Delta\right)^{T} P & -\gamma I & \left(D_{a}(\hat{\rho}, \rho) \Delta\right)^{T} \\
C_{a}(\hat{\rho}) & D_{a}(\hat{\rho}, \rho) \Delta & -\gamma I
\end{array}\right]<0} \\
& P=\left[\begin{array}{cc}
P_{a} & 0 \\
0 & P_{11}
\end{array}\right]>0,
\end{aligned}
$$

the inequality $\left\|\hat{f}_{v}-f_{v}\right\|<\gamma\left\|\xi_{a}\right\|$ is satisfied, where the scalar $\gamma$ represents an upper bound on the $\mathcal{L}_{2}$ gain.

Proof. From the bounded real lemma (BRL) [13], inequality (39) guarantees the $\mathcal{L}_{2}$ gain of the system (32) and (36) from $\xi_{a}(t, y, u)$ to $\hat{f}_{v}(t)-f_{v}(t)$ is less than $\gamma$.

Since by assumption the set $\Omega$ is compact, it can be enclosed by a polytope, and then by determining the vertices of the enclosing polytope, the system in (32) and (36) can be represented as a polytopic system, with vertices $\beta_{1}, \beta_{2}, \ldots, \beta_{n \beta}$, where $n_{\beta}=2^{r}$. Hence the LPV matrices in (32) and (36), $\left(A_{a}(\hat{\rho}), B_{a}(\hat{\rho}, \rho), C_{a}(\hat{\rho}), D_{a}(\hat{\rho}, \rho)\right)$, can be replaced by $\left(A_{a}\left(\beta_{i}\right), B_{a}\left(\beta_{i}\right), C_{a}\left(\beta_{i}\right), D_{a}\left(\beta_{i}\right)\right)$ [13]. A sufficient condition for (39) to hold is that the LMI given in 
(39) can be solved for all the vertices of the polytopic system with LPV system matrices $\left(A_{a}\left(\beta_{i}\right), B_{a}\left(\beta_{i}\right), C_{a}\left(\beta_{i}\right), D_{a}\left(\beta_{i}\right)\right)$ [20]. The polytopic version of (39) and (40) will be used to synthesize the gain $L$ (which specifies the sliding mode LPV observer in (8)-(9) and the weighting matrix $W$ ). Formally the optimization problem is:

Minimize $\gamma$ subject to (40) and

$$
\left[\begin{array}{ccc}
A_{a}^{\mathrm{T}}\left(\beta_{i}\right) P+P A_{a}\left(\beta_{i}\right) & P\left(B_{a}\left(\beta_{i}\right) \Delta\right) & C_{a}^{\mathrm{T}}\left(\beta_{i}\right) \\
\left(B_{a}\left(\beta_{i}\right) \Delta\right)^{\mathrm{T}} P & -\gamma I & \left(D_{a}\left(\beta_{i}\right) \Delta\right)^{\mathrm{T}} \\
C_{a}\left(\beta_{i}\right) & D_{a}\left(\beta_{i}\right) \Delta & -\gamma I
\end{array}\right]<0
$$

with respect to $P, L$ and $W$.

Remark 2: In what follows $B_{a}$ and $D_{a}$ will be scaled with a weighting matrix $\Delta=\operatorname{diag}\left(\delta_{1}, \delta_{2}, \delta_{3}, \delta_{4}\right)$, where $\delta_{1}=\operatorname{diag}\left(\delta_{1,1}, \ldots, \delta_{1, n}\right), \delta_{2}=\operatorname{diag}\left(\delta_{2,1}, \ldots, \delta_{2, m}\right), \delta_{3}=\operatorname{diag}\left(\delta_{3,1}, \ldots, \delta_{3, k}\right)$ and $\delta_{4}=\operatorname{diag}\left(\delta_{4,1}, \ldots, \delta_{4, p}\right)$, to introduce extra design freedom. The components $\delta_{1}, \ldots, \delta_{4}$ are design gains which can be used to trade-off and weight the different elements of uncertainty in (22).

Remark 3: In the case when $\hat{\rho}=\rho$, and in the absence of uncertainty, i.e. when $\xi(t, y, u) \equiv 0$ and when $d(t) \equiv 0$, it follows from (16) and (35) that $B_{a}(\hat{\rho}, \rho) \xi_{a}(t, y, u) \equiv 0$ and $D_{a}(\hat{\rho}, \rho) \xi_{a}(t, y, u) \equiv 0$. As a consequence, (32) collapses to

$$
\dot{e}_{a}(t)=A_{a}(\hat{\rho}) e_{a}(t)
$$

It follow from the top left sub-block of (39) that $A_{a}^{\mathrm{T}}(\hat{\rho}) P+P A_{a}(\hat{\rho})<0$ and so $e_{a}(t) \rightarrow 0$ asymptotically. Since $D_{a}(\hat{\rho}, \rho) \xi_{a}(t, y, u)=0$ and $\hat{f}_{v}(t)-f_{v}(t)=C_{a}(\hat{\rho}) e_{a}(t), \hat{f}_{v}(t) \rightarrow f_{v}(t)$ as $t \rightarrow \infty$, i.e. asymptotic fault estimation (and asymptotic state estimation) takes place. If either $\xi(t, y, u) \neq 0$ or $\hat{\rho} \neq \rho$, generically, asymptotic estimation is lost and the performance of the fault estimation is given in terms of the $\mathcal{L}_{2}$ gain $\gamma$ in Theorem 1. Provided the optimization in (41) yields a small value of $\gamma$, good estimation of $f_{v}(t)$ (in an $\mathcal{L}_{2}$ sense) is still achieved.

Finally, the estimate of the virtual fault $\hat{f}_{v}(t)$ is obtained from (31), and the actual fault can be recovered using (7) as

$$
\hat{f}_{i}(t)=E(\hat{\rho})^{-1} \hat{f}_{v}(t) .
$$

\section{ReCONSTRUCTION OF SENSOR FAULTS FOR UnCERTAin LPV Systems}

The problem of sensor fault reconstruction can be reformulated as an actuator fault reconstruction [20] which allows the sliding mode observer scheme for actuator fault reconstruction given in Section III to be employed. Consider an uncertain LPV system subject to sensor faults given by

$$
\begin{aligned}
\dot{x}(t) & =A(\rho) x(t)+B(\rho) u(t)+M \xi(t, y, u) \\
y(t) & =C x(t)+N f_{o}(t)+d(t),
\end{aligned}
$$

where $f_{o} \in \mathbb{R}^{r}$ models the unknown sensor faults and the fixed matrix $N \in \mathbb{R}^{p \times r}$ is known. It is assumed that $r<p$. The remaining matrices and vectors in (44) and (45) are assumed to have the same properties (and dimensions) as those in (1) and (2). By scaling and rearranging the outputs, if necessary, the output vector can be written as

$$
y(t)=\left[\frac{y_{1}(t)}{y_{2}(t)}\right]=\left[\frac{C_{1}}{C_{2}}\right] x(t)+\left[\frac{0}{I_{r}}\right] f_{o}(t)+\left[\frac{d_{1}(t)}{d_{2}(t)}\right],
$$

where the fixed matrices $C_{1} \in \mathbb{R}^{(p-r) \times n}, C_{2} \in \mathbb{R}^{r \times n}$. It is assumed that $y_{1}(t) \in \mathbb{R}^{p-r}$ are fault free outputs and $y_{2}(t) \in \mathbb{R}^{r}$ are potentially prone to faults. As in [20] consider a new state $z_{f}(t) \in \mathbb{R}^{r}$ constructed by passing $y_{2}(t)$ through a filter which satisfies the differential equation

$$
\dot{z}_{f}(t)=-A_{f} z_{f}(t)+A_{f} y_{2}(t)
$$

where $A_{f} \in \mathbb{R}^{r \times r}$ is a stable Hurwitz matrix.

A new state vector can be formed by augmenting $x(t)$ from (44) with $z_{f}(t)$ (obtained by substituting $y_{2}(t)$ from (46) into (47)). The new state vector $x_{s}(t)=\operatorname{col}\left(x(t), z_{f}(t)\right)$ and its derivative satisfies

$$
\dot{x}_{s}(t)=A_{s}(\rho) x_{s}(t)+B_{s}(\rho) u(t)+H_{s} f_{o}(t)+M_{s} \xi_{s}(t, y, u)
$$


where $\xi_{s}(t, y, u)=\operatorname{col}\left(\xi(t, y, u), d_{2}(t)\right)$ and the matrices

$$
A_{s}(\rho)=\left[\begin{array}{cc}
A(\rho) & 0 \\
A_{f} C_{2} & -A_{f}
\end{array}\right], \quad B_{s}(\rho)=\left[\begin{array}{c}
B(\rho) \\
0
\end{array}\right], \quad H_{s}=\left[\begin{array}{c}
0 \\
A_{f}
\end{array}\right], \quad \text { and } \quad M_{s}=\left[\begin{array}{cc}
M & 0 \\
0 & A_{f}
\end{array}\right] .
$$

A new output vector can be constructed by augmenting $y_{1}(t)$ from (46) and $z_{f}(t)$ from (47), $y_{s}(t)=\operatorname{col}\left(y_{1}(t), z_{f}(t)\right)$, such that

$$
y_{s}(t)=C_{s}(t) x_{s}(t)+d_{s}(t)
$$

where the matrices

$$
C_{s}=\left[\begin{array}{cc}
C_{1} & 0 \\
0 & I_{r}
\end{array}\right] \quad \text { and } \quad d_{s}(t)=\left[\begin{array}{c}
d_{1}(t) \\
0
\end{array}\right]
$$

The augmented system in (48) and (49) has a similar form to the system given in (1) and (2), and therefore the fault reconstruction scheme given in Section III can be directly used to reconstruct the sensor fault $f_{o}(t)$.

\section{RECONFIGURE BENCHMARK MODEL}

The Reconfiguration of Control in Flight for Integral Global Upset Recovery (RECONFIGURE) project aims to study fault tolerant scenarios for a passenger aircraft. The benchmark mainly consists of a high-fidelity nonlinear aircraft model which is used to simulate nominal flight conditions and realistic fault scenarios [34]. The validated aircraft model has been delivered to the consortium by Airbus to explore various fault tolerant mechanisms. The benchmark model covers the complete nonlinear flight domain along with the sensors, actuators, flight control computers, guidance and navigation mechanisms, etc. [34]. Several linear models (which covers a limited operating range) at various operating points were provided by AIRBUS. An LPV model was built based on the one described in [35] to explore the performance of the fault detection and reconstruction scheme given earlier in this paper. The designed LPV SMO is validated on the high-fidelity nonlinear aircraft model for the complete operating envelope. Here a sensor fault scenario is considered for the RECONFIGURE benchmark problem and used to test the proposed FDI scheme.

\section{A. LPV models}

An LPV model of the RECONFIGURE benchmark problem has been developed in [35]. The state vector comprises pitch rate (deg/s), ground speed (kts), ground angle of attack (deg) and pitch angle (deg), i.e. $\left[\begin{array}{llll}V_{g} & \alpha & \theta\end{array}\right]^{\mathrm{T}}$; the plant inputs are the left inboard elevator, right inboard elevator, left outboard elevator, right outboard elevator and trimmable horizontal stabiliser, $\left[\begin{array}{lllll}\delta_{e i l} & \delta_{e i r} & \delta_{e o l} & \delta_{e o r} & \delta_{s t a b}\end{array}\right]^{\mathrm{T}}$. In this paper it is assumed the all the states are measured and hence the outputs and the states are the same. The scheduling parameters for the LPV model are: mass $w$ (tons), x-position of the center of gravity $c . g .(\% / 100)$, conventional airspeed $V_{c a s}(\mathrm{~m} / \mathrm{s})$, and the Mach number Mach; $\rho:=\left[\begin{array}{llll}w & c . g . & V_{\text {cas }} & M a c h\end{array}\right]$.

\section{B. Design}

This paper considers the longitudinal axes of the aircraft. The relevant control problems in the RECONFIGURE benchmark are concerned with maintaining the normal load factor and angle of attack protection control laws. The specific objective here is to reconstruct sensor faults in $\alpha$ and $V_{g}$ using a sliding mode observer with imperfectly known scheduling parameters. In this paper the scheduling parameters, $\rho$, is assumed to be known only up to a certain degree of accuracy and in particular that conventional airspeed $V_{\text {cas }}$ is subject to a fault. For the design, the states of the LPV system are:

$$
x=\left[\begin{array}{llll}
\theta & q & \alpha & V_{g}
\end{array}\right]^{\mathrm{T}} .
$$

A new state vector $x_{s}$ is created by augmenting $x$ with the $z_{f}=\left[\begin{array}{ll}\alpha_{f} & V_{g_{f}}\end{array}\right]^{\mathrm{T}}$ obtained by passing the measurements of $\alpha$ and $V_{g}$ through a filter which satisfies (47). Here $A_{f}$ is chosen as the second order identity matrix. The augmented state vector is given by

$$
x_{s}=\left[\begin{array}{llllll}
\theta & q & \alpha & V_{g} & \alpha_{f} & V_{g_{f}}
\end{array}\right]^{\mathrm{T}} .
$$

A new output vector for driving the observer is formed by augmenting fault free outputs with $z_{f}$, so that

$$
y_{s}=\left[\begin{array}{llll}
\theta & q & \alpha_{f} & V_{g_{f}}
\end{array}\right]^{\mathrm{T}} .
$$


The LPV matrices have an affine representation

$$
\begin{aligned}
& A(\rho)=A_{0}+\sum_{i=1}^{4} \rho_{i} A_{i} \\
& B(\rho)=B_{0}+\sum_{i=1}^{4} \rho_{i} B_{i}
\end{aligned}
$$

The elevators are assumed to move in tandem and hence they have been aggregated to create a single elevator input $\delta_{e}$. The corresponding input distribution matrix $B_{s}(\rho)$ in (48), based on the state vector in (50), has the form $B_{s}(\rho)=\left[\begin{array}{ll}B(\rho) & 0\end{array}\right]^{\mathrm{T}}$, where (true) plant input distribution matrix

$$
B(\rho)=\left[\begin{array}{llll}
B_{11}(\rho) & B_{21}(\rho) & 0 & B_{41}(\rho) \\
B_{12}(\rho) & B_{22}(\rho) & 0 & B_{42}(\rho)
\end{array}\right]^{\mathrm{T}} .
$$

Permutating the states of the system in (51) yields

$$
x_{s}=\left[\begin{array}{lllllll}
\alpha & V_{g} & \theta & q & \alpha_{f} & V_{g_{f}}
\end{array}\right]^{\mathrm{T}},
$$

The derivative of $x_{s}$ satisfies

$$
\dot{x}_{s}(t)=A_{s}(\rho) x_{s}(t)+B_{s}(\rho) u(t)+H_{s} f_{o}(t)+M_{s} \xi_{s}(t, y, u),
$$

where $H_{s}=\left[\begin{array}{ll}0 & A_{f}\end{array}\right]^{\mathrm{T}}$. Since, $\alpha$ and $V_{g}$ are prone to faults, the corresponding elements of the uncertainty distribution matrix, $M_{s}=\left[\begin{array}{cc}M & 0 \\ 0 & A_{f}\end{array}\right]$, has been given more weights such that

$$
M=\left[\begin{array}{cccc}
0.01 & 0 & 0 & 0 \\
0 & 0.01 & 0 & 0
\end{array}\right]^{\mathrm{T}} .
$$

The outputs (as the function of transformed states in (54)) can be written as

$$
y_{s}=\left[\begin{array}{ll|llll}
0 & 0 & 1 & 0 & 0 & 0 \\
0 & 0 & 0 & 1 & 0 & 0 \\
0 & 0 & 0 & 0 & 1 & 0 \\
0 & 0 & 0 & 0 & 0 & 1
\end{array}\right] x_{s}=\left[\begin{array}{ll}
0 & I_{4}
\end{array}\right] x_{s}
$$

The transformed system in (54) and (56) are in the form of (7) and (5). Hence the LPV SMO given in section III can be synthesised to estimate the sensor faults. It is assumed that the fault estimation is equally affected by the uncertainty in the state vector, control input (due to imperfect knowledge of $\rho$ ), plant uncertainty $\xi(t)$, and measurement corruption $\phi(t)$. Here the parameters of the design weighting matrix $\Delta$ in (39) are chosen as $\delta_{1}=\operatorname{diag}(0.01,0.01,0.01,0.01,0.01,0.01), \delta_{2}=\operatorname{diag}(0.01,0.01), \delta_{3}=\operatorname{diag}(0.01,0.01,0.01,0.01)$ and $\delta_{4}=$ $\operatorname{diag}(0.01,0.01,0.01,0.01)$. These weights are used to scale the plant matrix, input matrix, output matrix and uncertainty matrix.

The gain $L$ in (15) has been synthesised by solving the LMIs in (39) in Matlab where the upper bound on $\mathcal{L}_{2}$ gain obtained is $\gamma=0.1728$. This gain is then used to evaluate $G_{n}$ in (14) and $G_{l}(\rho)=G_{l 0}+G_{l 1}\left(\hat{\rho}_{1}\right)+G_{l 2}\left(\hat{\rho}_{2}\right)+$ $G_{l 3}\left(\hat{\rho}_{3}\right)+G_{l 4}\left(\hat{\rho}_{4}\right)$ is evaluated from (25). Here the designer selected Hurwitz matrix $\tilde{A}_{22}^{s}$ which forms part of $G_{l}(\hat{\rho})$ has been chosen as $\operatorname{diag}(-3.33,-6.66,-3.33,-3.33)$. Finally, the faults are reconstructed from $(31)$, wherein the discontinuous function has been approximated as

$$
\nu_{e q}(t)=-\lambda(t, y, u, \hat{\rho}) \frac{P_{o} e_{y}(t)}{\left\|P_{o} e_{y}(t)\right\|+\delta},
$$

where $P_{o}$ is a Lyapunov matrix for $\tilde{A}_{22}^{s}$ and $\delta$ is a small positive constant. Finally, $\lambda$ and $\delta$ are chosen as $\operatorname{diag}(100,100,300,300)$ and 0.01 , respectively. 


\begin{tabular}{|c|c|c|c|c|c|c|c|c|c|c|c|c|c|c|c|c|c|c|c|c|c|}
\hline FP & 1 & 2 & 3 & 4 & 5 & 6 & 7 & 8 & 9 & 10 & 11 & 12 & 13 & 14 & 15 & 16 & 17 & 18 & 19 & wt (tons) & $\mathrm{cg}(\%)$ \\
\hline A & 1 & 10 & 19 & 31 & 43 & 55 & 67 & 79 & 91 & 103 & 115 & 127 & 139 & 151 & 163 & 175 & 187 & 199 & 211 & MFW & max. fwd \\
\hline в & 2 & 11 & 20 & 32 & 44 & 56 & 68 & 80 & 92 & 104 & 116 & 128 & 140 & 152 & 164 & 176 & 188 & 200 & 212 & MFW & med. \\
\hline c & 3 & 12 & 21 & 33 & 45 & 57 & 69 & 81 & 93 & 105 & 117 & 129 & 141 & 153 & 165 & 177 & 189 & 201 & 213 & MFW & max. aft \\
\hline D & 4 & 13 & 22 & 34 & 46 & 58 & 70 & 82 & 94 & 106 & 118 & 130 & 142 & 154 & 166 & 178 & 190 & 202 & 214 & $(M L W+M F Z W) / 2$ & max. fwd \\
\hline E & 5 & 14 & 23 & 35 & 47 & 59 & 71 & 83 & 95 & 107 & 119 & 131 & 143 & 155 & 167 & 179 & 191 & 203 & 215 & $(M L W+M F Z W) / 2$ & med. \\
\hline $\mathrm{F}$ & 6 & 15 & 24 & 36 & 48 & 60 & 72 & 84 & 96 & 108 & 120 & 132 & 144 & 156 & 168 & 180 & 192 & 204 & 216 & $(M L W+M F Z W) / 2$ & max. aft \\
\hline G & 7 & 16 & 25 & 37 & 49 & 61 & 73 & 85 & 97 & 109 & 121 & 133 & 145 & 157 & 169 & 181 & 193 & 205 & 217 & (MTOW+MFZW)/2 & max. fwd \\
\hline H & 8 & 17 & 26 & 38 & 50 & 62 & 74 & 86 & 98 & 110 & 122 & 134 & 146 & 158 & 170 & 182 & 194 & 206 & 218 & (MTOW+MFZW)/2 & med. \\
\hline 1 & 9 & 18 & 27 & 39 & 51 & 63 & 75 & 87 & 99 & 111 & 123 & 135 & 147 & 159 & 171 & 183 & 195 & 207 & 219 & (MTOW+MFZW)/2 & max. aft \\
\hline J & & & 28 & 40 & 52 & 64 & 76 & 88 & 100 & 112 & 124 & 136 & 148 & 160 & 172 & 184 & 196 & 208 & 220 & MTOW & max. fwd \\
\hline $\mathrm{k}$ & & & 29 & 41 & 53 & 65 & 77 & 89 & 101 & 113 & 125 & 137 & 149 & 161 & 173 & 185 & 197 & 209 & 221 & MTOW & med. \\
\hline $\mathrm{L}$ & & & 30 & 42 & 54 & 66 & 78 & 90 & 102 & 114 & 126 & 138 & 150 & 162 & 174 & 186 & 198 & 210 & 222 & MTOW & max. aft \\
\hline $\mathrm{S} / \mathrm{F}$ & 0 & 0 & 0 & 0 & 0 & 0 & 0 & 1 & 1 & 2 & 2 & 3 & 3 & 4 & 4 & 4 & 4 & 5 & 5 & & \\
\hline Gear & 0 & 0 & 0 & 0 & 0 & 0 & 0 & 0 & 0 & 0 & 0 & 0 & 0 & 0 & 0 & 1 & 1 & 1 & 1 & & \\
\hline Alt. $(m)$ & 13,106 & 13,106 & 9,144 & 9,144 & 4,572 & 4,572 & 4,572 & 2,286 & 2,286 & 2,286 & 2,286 & 1,524 & 1,524 & 610 & 610 & 305 & 305 & 305 & 305 & & \\
\hline Vcas $(\mathrm{m} / \mathrm{s})$ & VLS-5 & $\begin{array}{c}\mathrm{VMO} / \mathrm{M} \\
\mathrm{MO}\end{array}$ & vLS & vMO & vLS & VMO-90 & VMO & $\begin{array}{c}\text { (ARS+AES) } \\
/ 2\end{array}$ & VFE & vLS & \begin{tabular}{|c} 
(ARS+AES) \\
$/ 2$
\end{tabular} & vLS & VFE & VLS & VFE & VLS & VFE & VLS & VFE & & \\
\hline
\end{tabular}

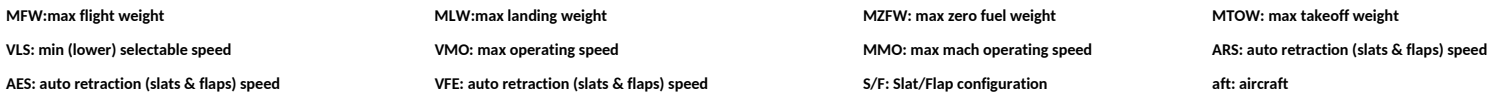

MZFW: max zero fuel weight

MMO: max mach operating speed S/F: Slat/Flap configuration

MTOW: max takeoff weight

ARS: auto retraction (slats \& flaps) speed aft: aircraft

TABLE I

FLIGHT OPERATING POINTS SHOWING THE LPV REGION (RED SHADE) AND COMPLETE FLIGHT ENVELOPE (WHITE SHADE)

\section{RECONFIGURE FES}

In this paper the FDI scheme will be tested during both static and dynamic manoeuvres for various parametric dispersions. The parametric dispersions include variations in weight (minimum flight weight to maximum take-off weight), altitude (varying from ground to ceiling altitude), calibrated speed (minimum selectable speed to maximum operating speed), slat/flap configurations (minimum to maximum slat/flap extensions), and with up and down landing gear positions. For the static flight case, the pilot stick command is held at 0 deg. For the dynamic manoeuvre the pilot stick command is a doublet signal which varies from $-1 \mathrm{deg}$ to $+1 \mathrm{deg}$. The simulation data for various operating conditions has been harvested from the functional engineering simulator (FES) [36] developed by the other industrial partner of the project, Deimos; and used to test the performance of the observer.

\section{Simulations}

An existing controller has been used to maintain the load factor at the required reference command [32]. The faults in $\alpha$ and $V_{g}$ are assumed to be (a) step signals, occurring at $10 \mathrm{~s}$; (b) oscillatory (sinusoid) signals occurring at $10 \mathrm{~s}$; (c) drift faults which starts from $10 \mathrm{~s}$. The simulations have been performed using the scheme proposed in the paper with imperfect scheduling parameters $(\hat{\rho})$. In all the simulations the observer has been implemented based on a $0.01 s$ sampling time. Note that the LPV observer design is based on the operating range given in Section V-A, but the simulation data it is tested on covers a much wider range.

1) Static manoeuvres: In this section, simulation results are shown when the pilot stick command is held at 0 deg.

a) Imperfect Scheduling parameters $(\hat{\rho})$ : In this subsection it is assumed that $\rho$ is prone to faults and is imperfectly measured. More specifically, the conventional airspeed, $V_{c a s}$, is prone to faults and is perturbed by $30 \%$ of its correct value. For the proposed SMO design the scheduling parameter set is $\hat{\rho}=\left[\begin{array}{llll}w & c . g . & 0.7 V_{\text {cas }} & \text { Mach }\end{array}\right]$. The pilot stick commands and the corresponding load factors are shown in Fig. 2(a). The zero pilot stick command translates to a load factor of $1 \mathrm{~g}$. The initial conditions for the aircraft states are shown in Fig. 2(b): the observer initial conditions are the same as that of the plant. The observer states and altitude of the aircraft are shown in Fig. 3(a). The elevator and stabiliser deflections are shown in Fig. 2(c); the elevators are trimmed at 0 deg, whereas the stabilisers are trimmed based on the corresponding operating points. Since, for the static manoeuvres the pilot stick is held at $0 \mathrm{deg}$, the control surfaces and the states are in steady state, except when the fault is injected in $V_{\text {cas }}$ and $\alpha$ at $10 \mathrm{~s}$. The results with imperfect scheduling parameters are shown in Fig. 2(d). Note that $V_{\text {cas }}$ in Fig. 2(d) is $70 \%$ of the true $V_{\text {cas }}$ value. The actual step faults (dotted line) and estimated step faults (solid lines), and the norm of the output estimation error using the proposed SMO with imperfect scheduling parameters are shown in 

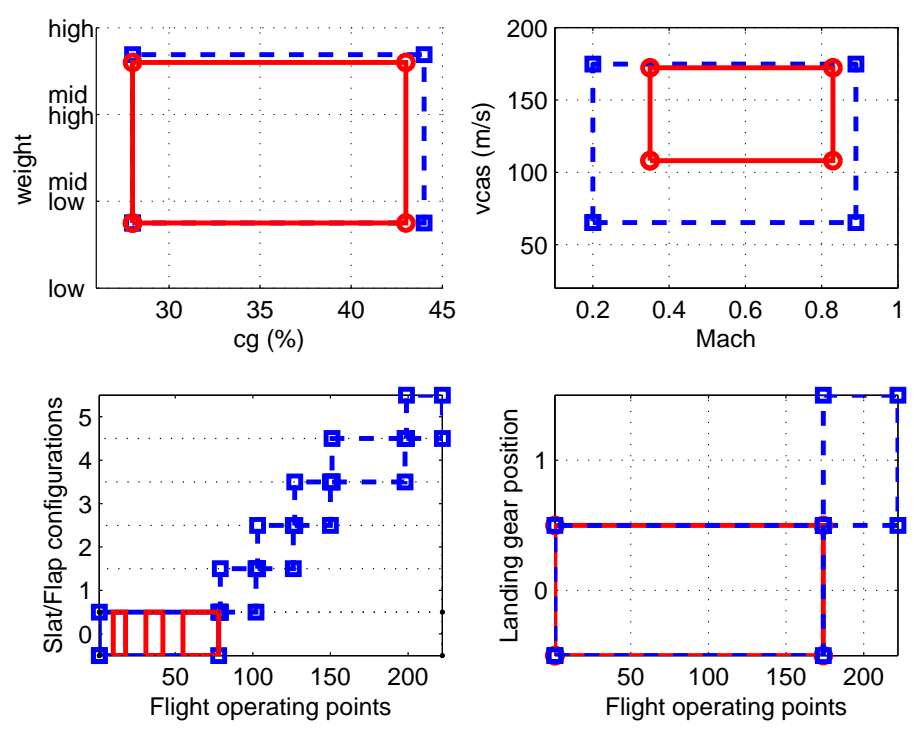

Fig. 1. Flight envelope; solid line represents the valid LPV region and dashed line represents the regions in which non-linear aircraft simulations are performed

Fig. 3(b). The proposed scheme has further been tested for oscillatory and drift faults. The states, in the presence of oscillatory and drift faults, are shown in Figs. 4(a) and 5(a) respectively. The corresponding fault reconstructions and the norm of the output estimation errors are shown in Figs. 4(b) and 5(b) respectively. The fault reconstruction errors for the step, oscillatory and drift faults are shown in Figs. 3(c), 4(c) and 5(c).

For all the three types of faults, the norm of the output estimation error is close to zero, which shows sliding is maintained in spite of the faults and imperfect scheduling parameters using the proposed SMO. The good performance of the proposed SMO, in the presence of imperfect scheduling parameters, is due to the fact that the uncertainty in $\rho$ has been considered at the design stage, and the SMO gains have been synthesised directly in such a way that the effect of $\hat{\rho}-\rho$ on the fault reconstruction is minimised.

2) Dynamic manoeuvres: In this section, simulation results are shown where the pilot stick command is a doublet signal which varies from -1 deg to +1 deg. Note that the LPV observer is designed based on an LPV model with a limited operating range as given in Section V-A, and covers only the highlighted operating points in Table 1; which comprises only a clean Slat/Flap (S/F) configuration, with zero landing gear position, and limited altitude and $V_{\text {cas }}$ range. However, the simulation results cover a much wider operating envelope (from near landing/takeoff (low altitude and speed) to cruise conditions (high speed and altitude)) consisting of all the 222 operating points shown in Table 1. The same can be seen in Fig. 1, where the solid line shows the LPV region for which the observer is designed, and the dashed line shows the operating range in which the observer performance is tested. Note that the exact value of $V_{\text {cas }}$, weight and c.g. in Table 1 are not provided due to industrial restrictions.

a) Imperfect Scheduling parameters $(\rho)$ : In this subsection it is assumed that the conventional airspeed, $V_{\text {cas }}$, is prone to faults and is perturbed by $30 \%$ of its correct value. Similar to the static manoeuvres case with imperfect scheduling parameters, here the scheduling parameter is set as $\hat{\rho}=\left[\begin{array}{llll}w & c . g . & 0.7 V_{\text {cas }} & \text { Mach }\end{array}\right]$. The pilot stick command and the corresponding load factor are shown in Fig. 6(a). The initial conditions for the aircraft states are shown in Fig. 6(b), and the observer initial conditions are the same as that of the plant. The observer states and altitude of the aircraft are shown in Fig. 7(a). Similar to the static manoeuvres case, step, oscillatory and drift faults are added to $V_{g}$ and $\alpha$. The elevator and stabiliser deflections are shown in Fig. 6(c); these control signals for various operating points are obtained from the existing AIRBUS controller [27] through the FES. The results with imperfect scheduling parameters, for dynamic manoeuvres, are shown in Fig. 6(d). The $V_{\text {cas }}$ in Fig. 6(d) is $70 \%$ of the perfect $V_{\text {cas }}$. The actual step faults (dotted line) and their estimates (solid lines), and the norm of the output estimation error using the proposed SMO with imperfect scheduling parameters are shown in Fig. 7(b). Similar to static manoeuvres case, the proposed scheme has further been tested for oscillatory and drift faults. The states, in the presence of oscillatory and drift faults, are shown in Figs. 8(a) and 9(a), respectively. The corresponding fault reconstructions and norms of the output estimation errors are shown in Figs. 8(b) and 9(b), respectively. The fault reconstruction errors for the step, oscillatory and drift faults are shown in Figs. 7(c), 8(c) and 9(c). Fault estimation 
is retained due to the fact that the uncertainty in $\rho$ has been considered at the design stage and the SMO gains have been synthesised directly in such a way that the effect of $\hat{\rho}-\rho$ on the fault reconstruction is minimised. The faults reconstructions for the dynamic manoeuvres are slightly degraded compared to the static manoeuvres case; this is due to the fact that the operating range for dynamic manoeuvres is much wider than the LPV region where the proposed observer is built.

\section{Vi. CONCLUSions}

In this paper, a sliding mode observer has been proposed with the aim of reconstructing actuator and sensor faults for a class of linear parameter varying systems in the presence of imperfect knowledge of the scheduling parameters. The observer gains are synthesised to minimise the effect of uncertainties, imperfect scheduling parameters and measurement corruptions on the fault reconstruction in an $\mathcal{L}_{2}$ gain sense. For the actuator fault case, the virtual fault is first reconstructed and then an estimate of the actual fault is recovered from it; whereas a sensor fault is directly reconstructed. The efficacy of the proposed approach has been validated based on simulations and data from the high fidelity RECONFIGURE benchmark model for sensor fault reconstruction. The proposed observer shows good reconstruction of the sensor faults in the presence of correct and imperfect scheduling parameters.

\section{ACKNOWLEDGEMENTS}

This work is supported by the EU-FP7 Grant (FP7-AAT-2012-314544): RECONFIGURE.

\section{REFERENCES}

[1] J. Chen, R. J. Patton, Robust model-based fault diagnosis for dynamic systems, Springer, 2012.

[2] P. Lu, L. Van Eykeren, E.-J. van Kampen, C. de Visser, Q. Chu, Double-model adaptive fault detection and diagnosis applied to real flight data, Control Engineering Practice 36 (2015) 39-57.

[3] P. Lu, L. Van Eykeren, E. van Kampen, C. de Visser, Q. Chu, Aircraft inertial measurement unit fault identification with application to real flight data, Journal of Guidance, Control, and Dynamics 38 (12) (2015) 2467-2475.

[4] A. Marcos, S. Ganguli, G. J. Balas, An application of $H_{\infty}$ fault detection and isolation to a transport aircraft, Control Engineering Practice 13 (1) (2005) 105-119.

[5] I. Yaesh, U. Shaked, Robust $H_{\infty}$ deconvolution and its application to fault detection, Journal of Guidance, Control, and Dynamics 23 (6) (2000) 1001-1012.

[6] D. Henry, J. Cieslak, A. Zolghadri, D. Efimov, A non-conservative $H_{-} / H \infty$ solution for early and robust fault diagnosis in aircraft control surface servo-loops, Control Engineering Practice 31 (2014) 183-199.

[7] N. Viswanadham, R. Srichander, Fault detection using unknown input observers, Control Theory and Advanced Technology 3 (2) (1987) 91-101.

[8] Y. Park, J. L. Stein, Closed-loop, state and input observer for systems with unknown inputs, International Journal of Control 48 (3) (1988) 1121-1136.

[9] D. Rotondo, M. Witczak, V. Puig, F. Nejjari, M. Pazera, Robust unknown input observer for state and fault estimation in discrete-time takagisugeno systems, International Journal of Systems Science 47 (2016) 3409-3424.

[10] C. Edwards, S. K. Spurgeon, R. J. Patton, Sliding mode observers for fault detection and isolation, Automatica 36 (4) (2000) $541-553$.

[11] H. Alwi, C. Edwards, C. P. Tan, Fault detection and fault-tolerant control using sliding modes, Springer, 2011.

[12] C. P. Tan, C. Edwards, Sliding mode observers for robust detection and reconstruction of actuator and sensor faults, International Journal of Robust and Nonlinear Control 13 (5) (2003) 443-463.

[13] P. Apkarian, P. Gahinet, A convex characterization of gain-scheduled $H_{\infty}$ controllers, IEEE Transactions on Automatic Control 40 (5) (1995) 853-864.

[14] J. S. Shamma, J. R. Cloutier, Gain-scheduled missile autopilot design using linear parameter varying transformations, Journal of Guidance, Control, and Dynamics 16 (2) (1993) 256-263.

[15] A. Varga, D. Ossmann, LPV model-based robust diagnosis of flight actuator faults, Control Engineering Practice 31 (2014) $135-147$.

[16] B. Vanek, A. Edelmayer, Z. Szab, J. Bokor, Bridging the gap between theory and practice in LPV fault detection for flight control actuators, Control Engineering Practice 31 (2014) 171-182.

[17] H. Alwi, C. Edwards, Development and application of sliding mode LPV fault reconstruction schemes for the ADDSAFE benchmark, Control Engineering Practice 31 (2014) 148-170.

[18] D. Efimov, L. Fridman, T. Raissi, A. Zolghadri, Interval estimation for LPV systems applying high order sliding mode techniques, Automatica 48 (9) (2012) 2365-2371.

[19] H. Alwi, C. Edwards, A. Marcos, Fault reconstruction using a LPV sliding mode observer for a class of LPV systems, Journal of the Franklin Institute 349 (2) (2012) 510-530.

[20] H. Alwi, C. Edwards, Robust fault reconstruction for linear parameter varying systems using sliding mode observers, International Journal of Robust and Nonlinear Control 24 (14) (2013) 1947-1968.

[21] G. Millérioux, L. Rosier, G. Bloch, J. Daafouz, Bounded state reconstruction error for LPV systems with estimated parameters, IEEE Transactions on Automatic Control 49 (8) (2004) 1385-1389.

[22] J. Daafouz, J. Bernussou, J. C. Geromel, On inexact LPV control design of continuous-time polytopic systems, IEEE Transactions on Automatic Control 53 (7) (2008) 1674-1678.

[23] W. H. Heemels, J. Daafouz, G. Millerioux, Observer-based control of discrete-time LPV systems with uncertain parameters, IEEE Transactions on Automatic Control 55 (9) (2010) 2130-2135. 

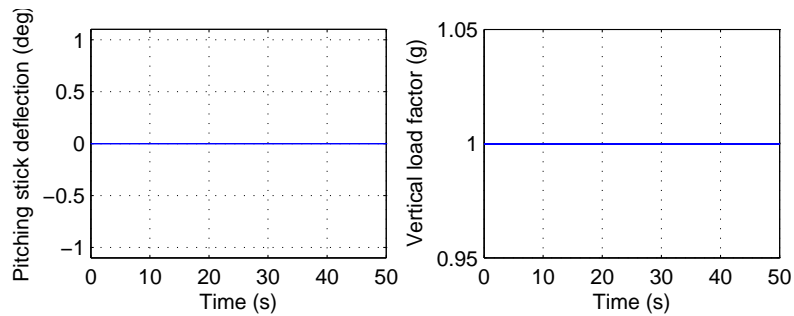

(a) Pilot stick command and the load factor
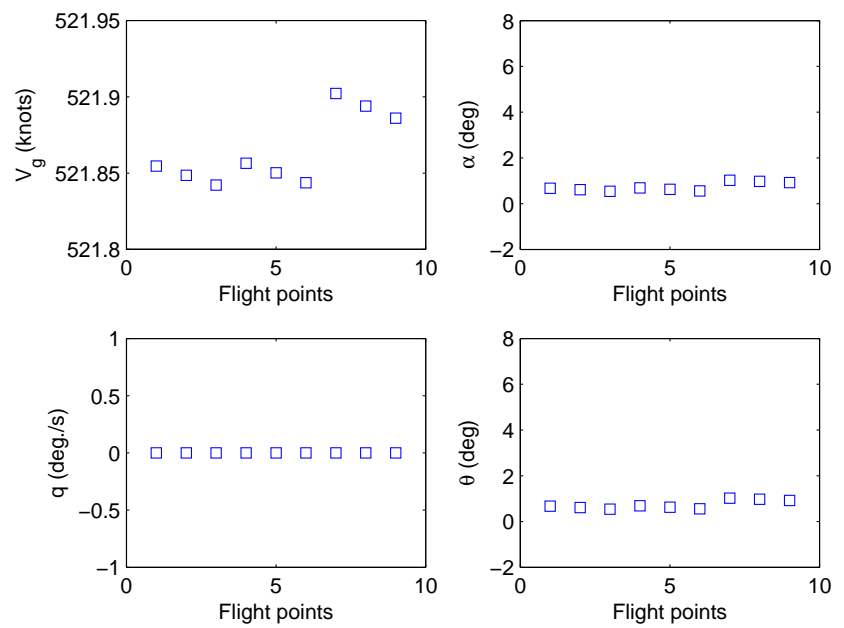

(b) States initial conditions
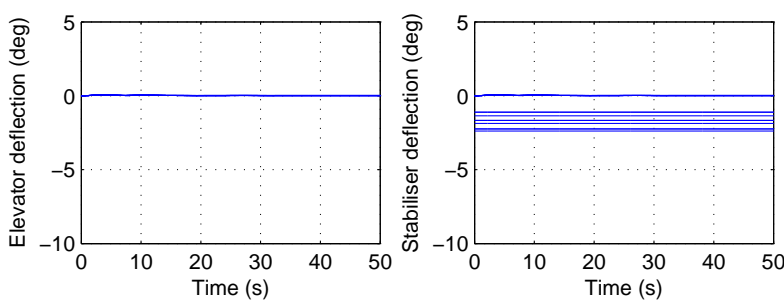

(c) Control surfaces
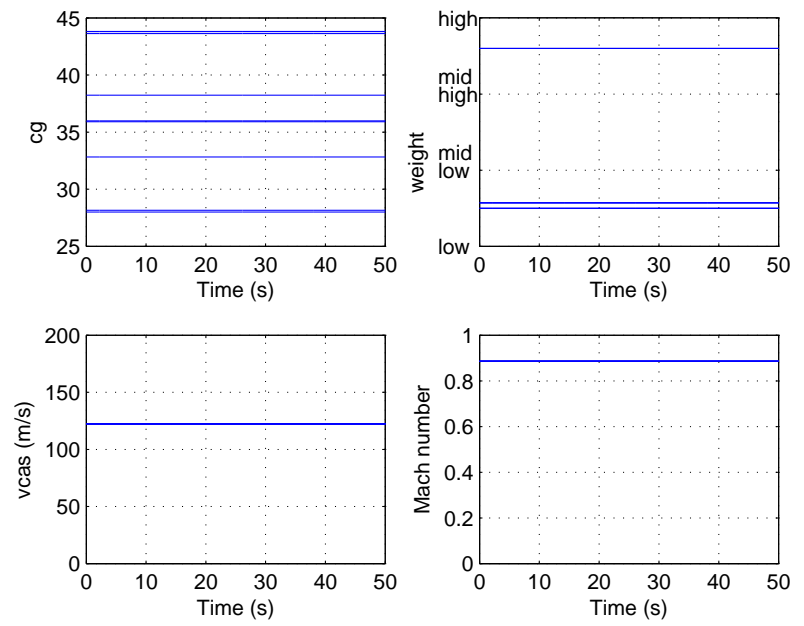

(d) Imperfect scheduling parameters

Fig. 2. Static manoeuvres: stepped faults with imperfect scheduling parameters (1 of 2$)$ 

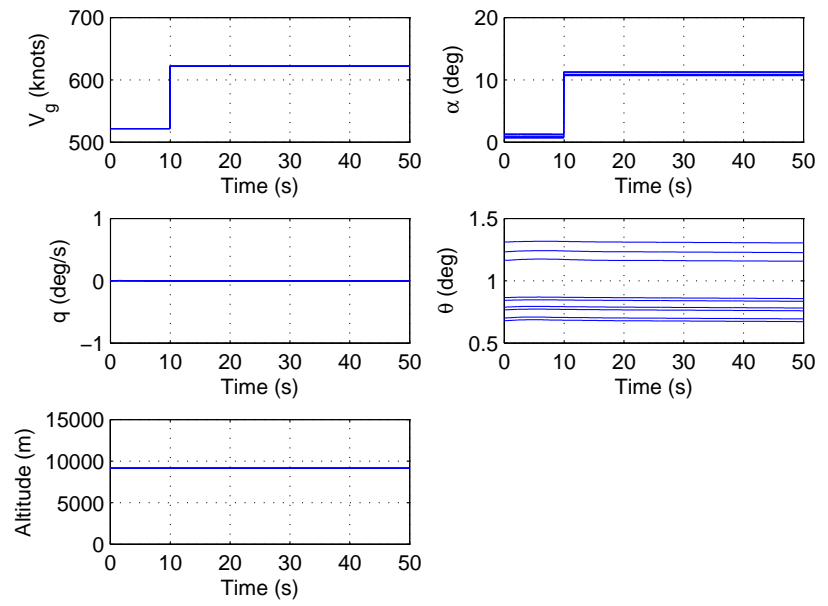

(a) Observer states and altitude
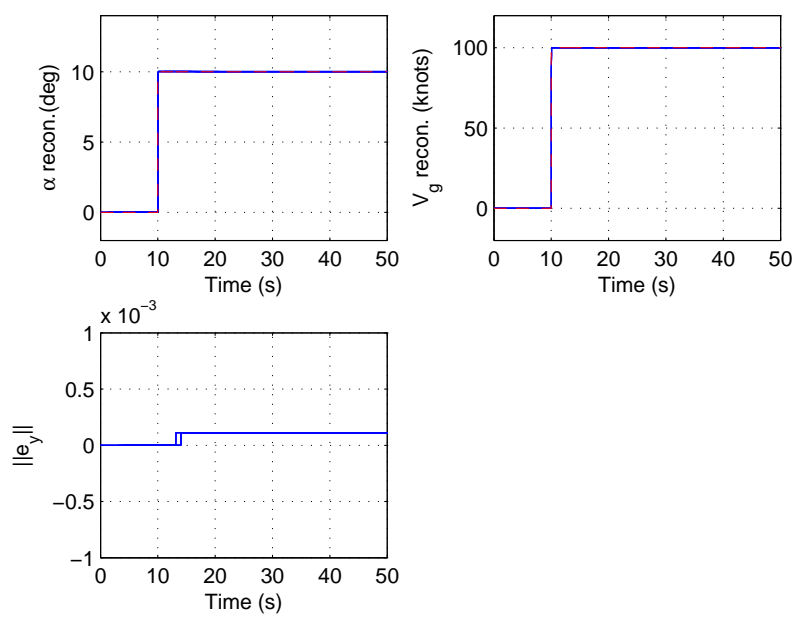

(b) Fault reconstruction (actual (dotted lines) and reconstructed (solid lines) faults) and norm of output error
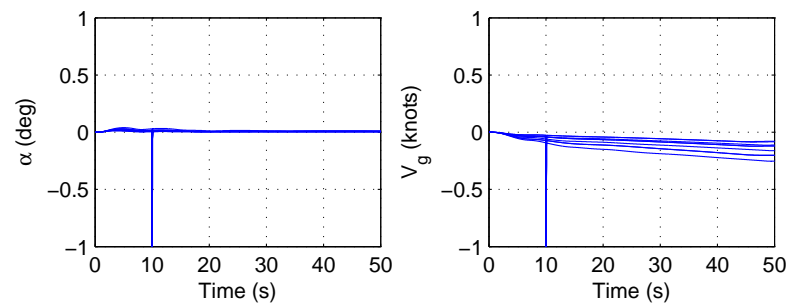

(c) Estimation errors

Fig. 3. Static manoeuvres: stepped faults with imperfect scheduling parameters (2 of 2) 

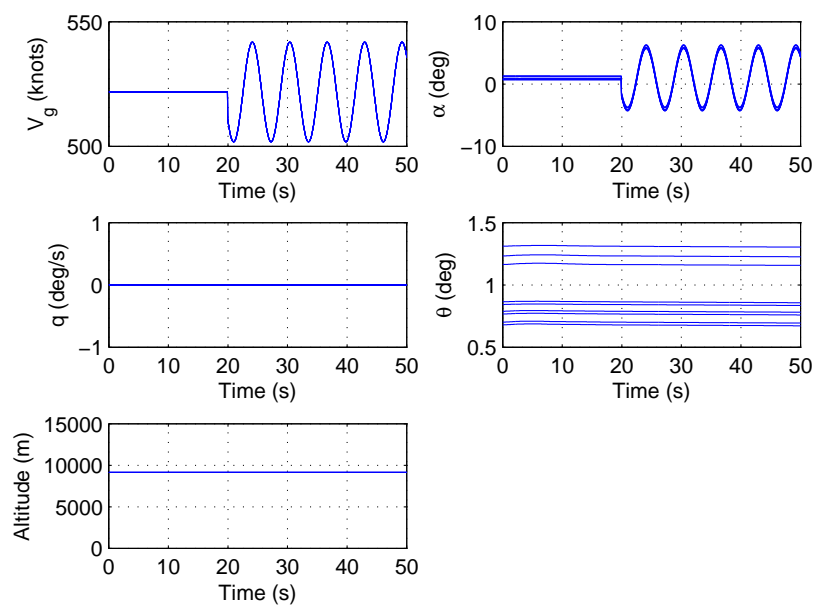

(a) Observer states and altitude
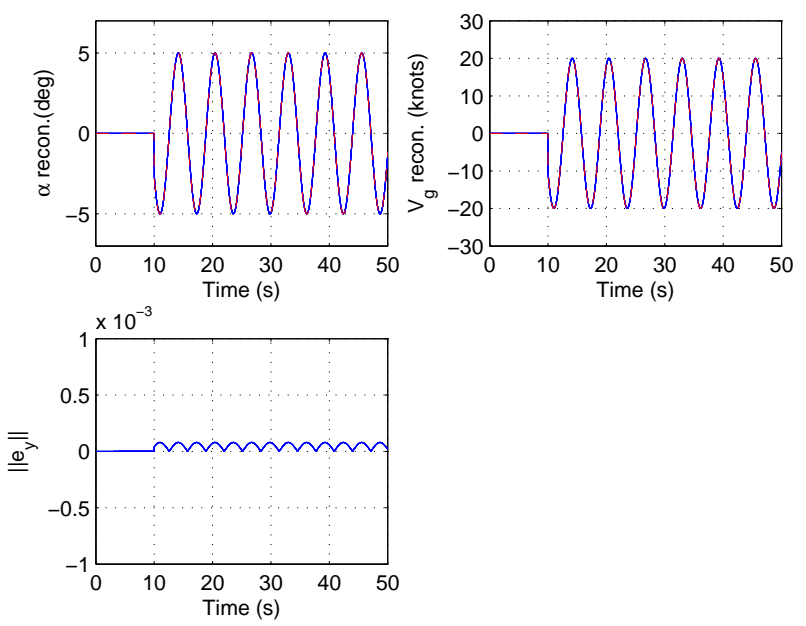

(b) Fault reconstruction (actual (dotted lines) and reconstructed (solid lines) faults) and norm of output error
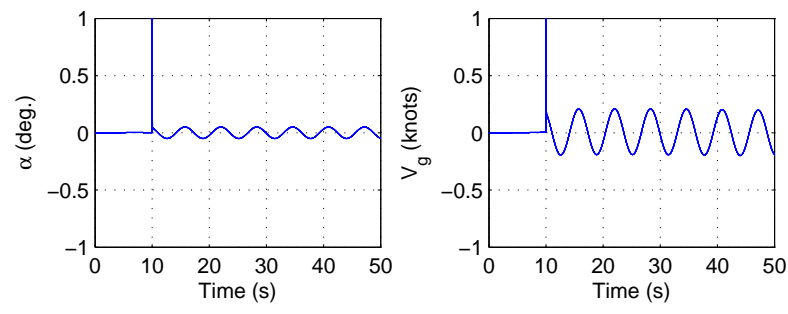

(c) Estimation errors

Fig. 4. Static manoeuvres: oscillatory faults with imperfect scheduling parameters 

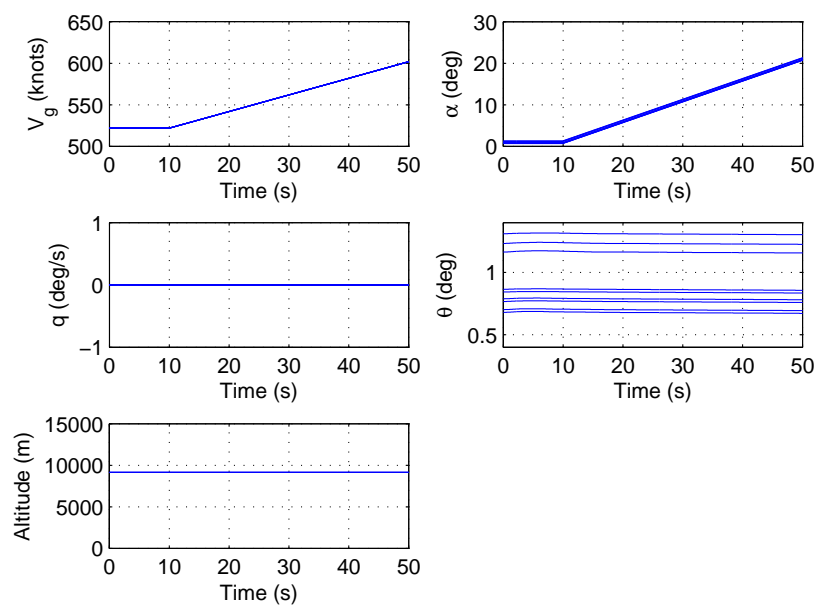

(a) Observer states and altitude
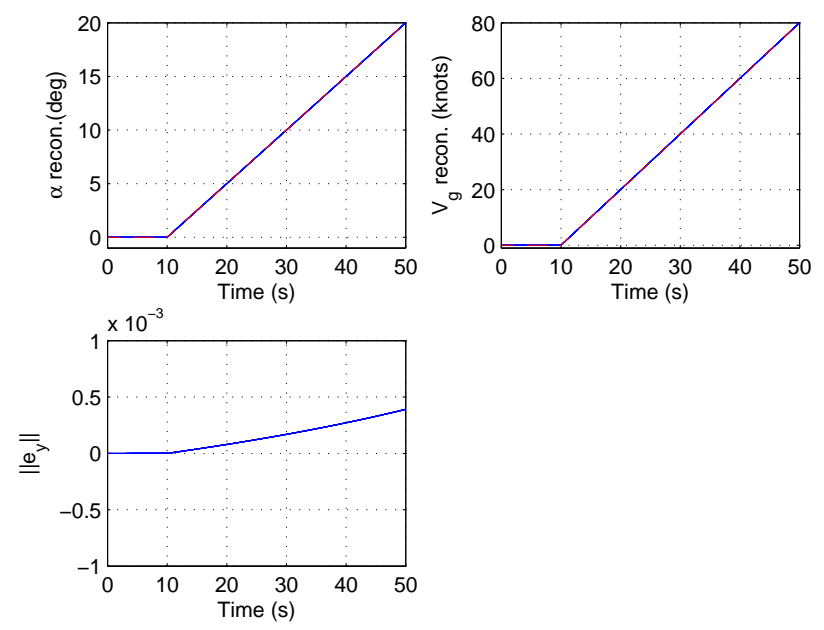

(b) Fault reconstruction (actual (dotted lines) and reconstructed (solid lines) faults) and norm of output error

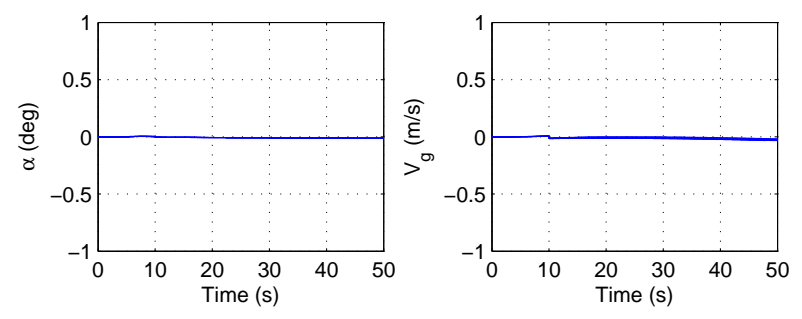

(c) Estimation errors

Fig. 5. Static manoeuvres: ramp faults with imperfect scheduling parameters 

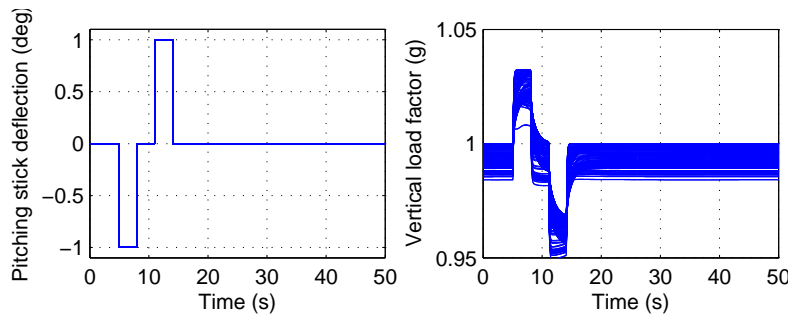

(a) Pilot stick command and the load factor
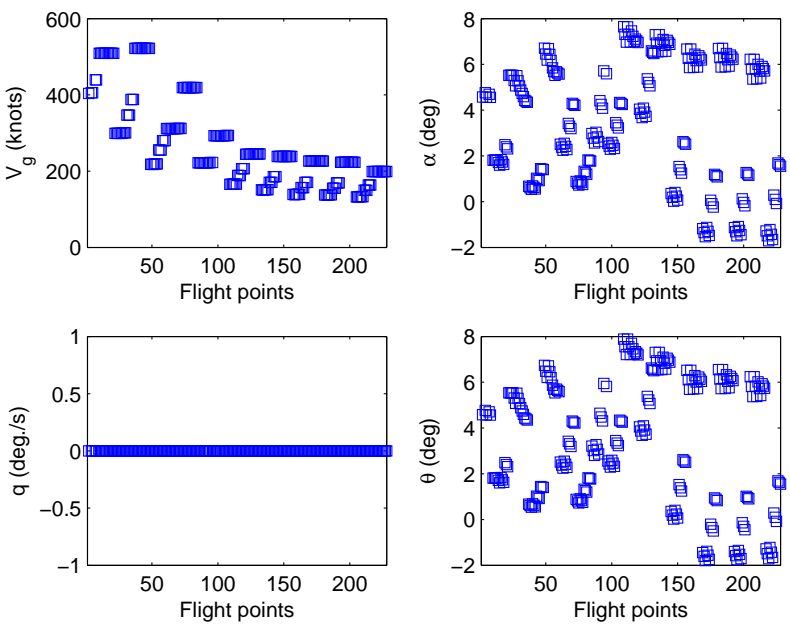

(b) States initial conditions
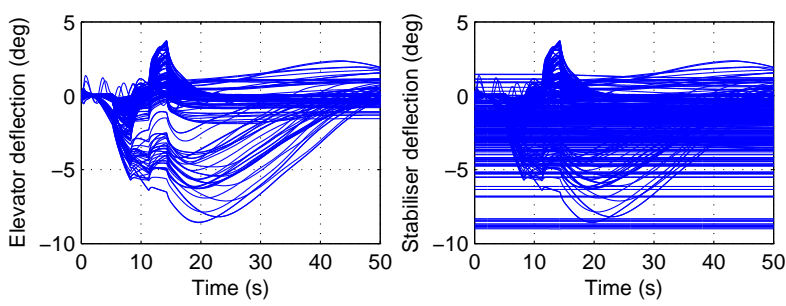

(c) Control surfaces
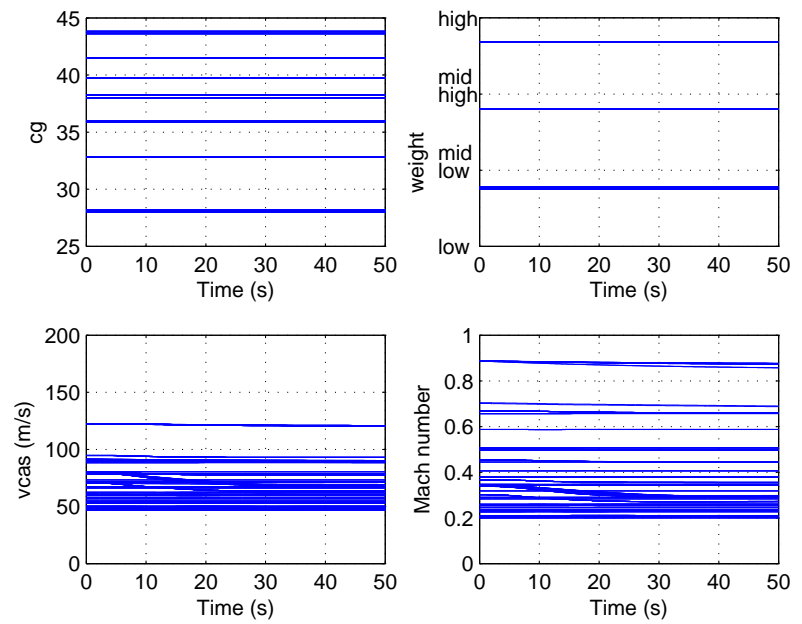

(d) Imperfect scheduling parameters

Fig. 6. Dynamic manoeuvres: stepped faults with imperfect scheduling parameters (1 of 2) 

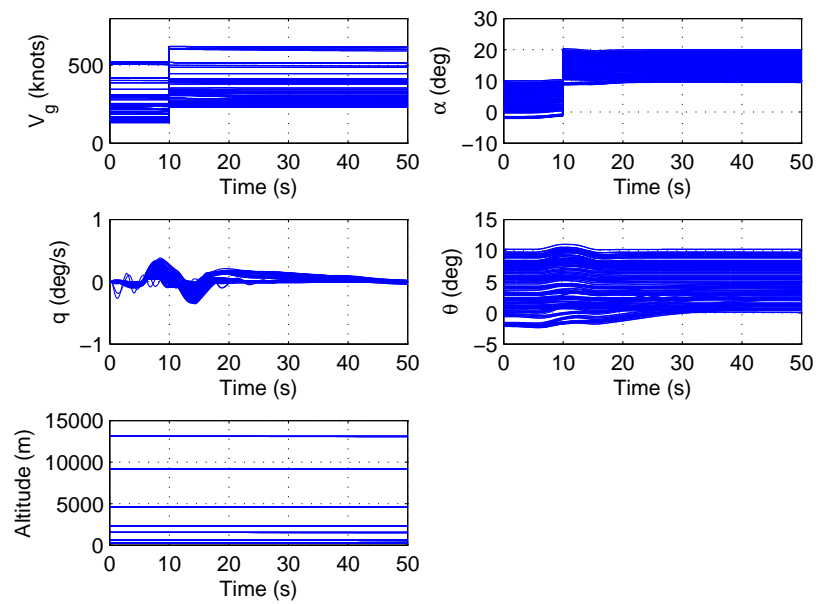

(a) Observer states and altitude
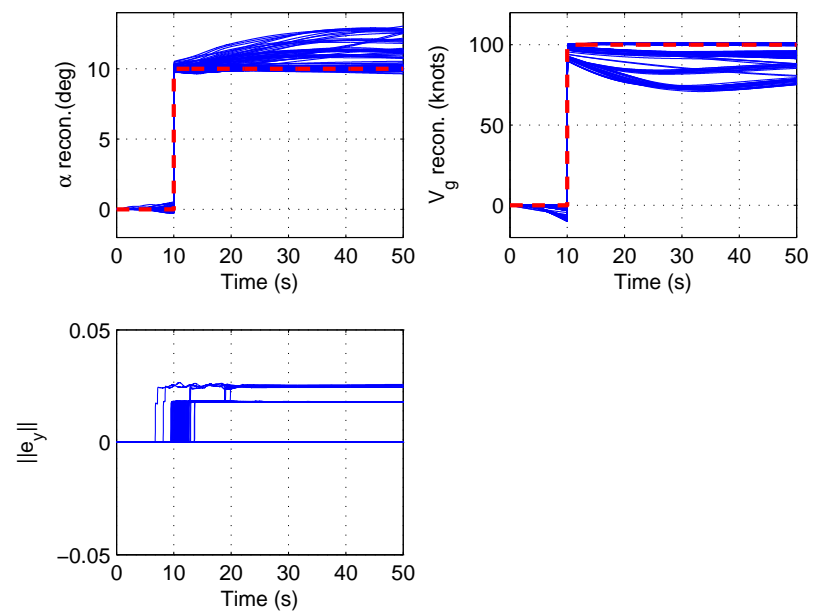

(b) Fault reconstruction (actual (dotted lines) and reconstructed (solid lines) faults) and norm of output error

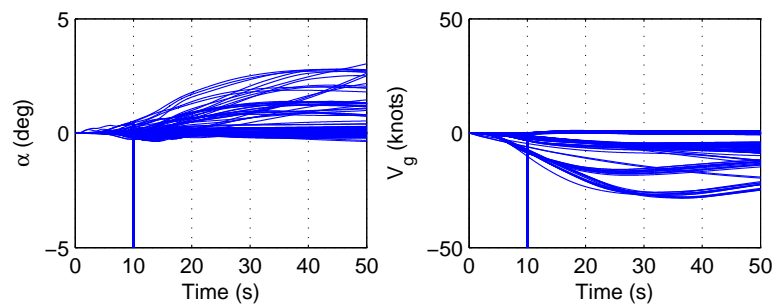

(c) Estimation errors

Fig. 7. Dynamic manoeuvres: stepped faults with imperfect scheduling parameters (2 of 2$)$ 

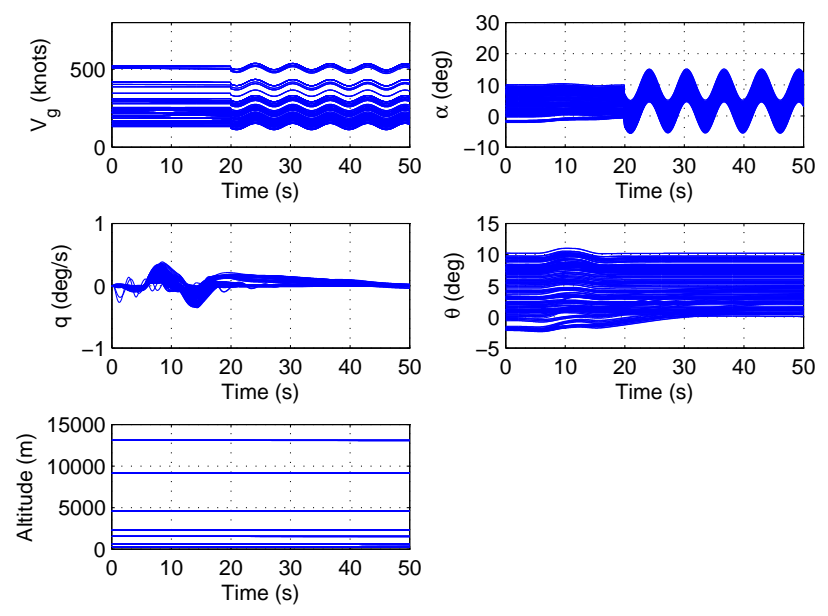

(a) Observer states and altitude
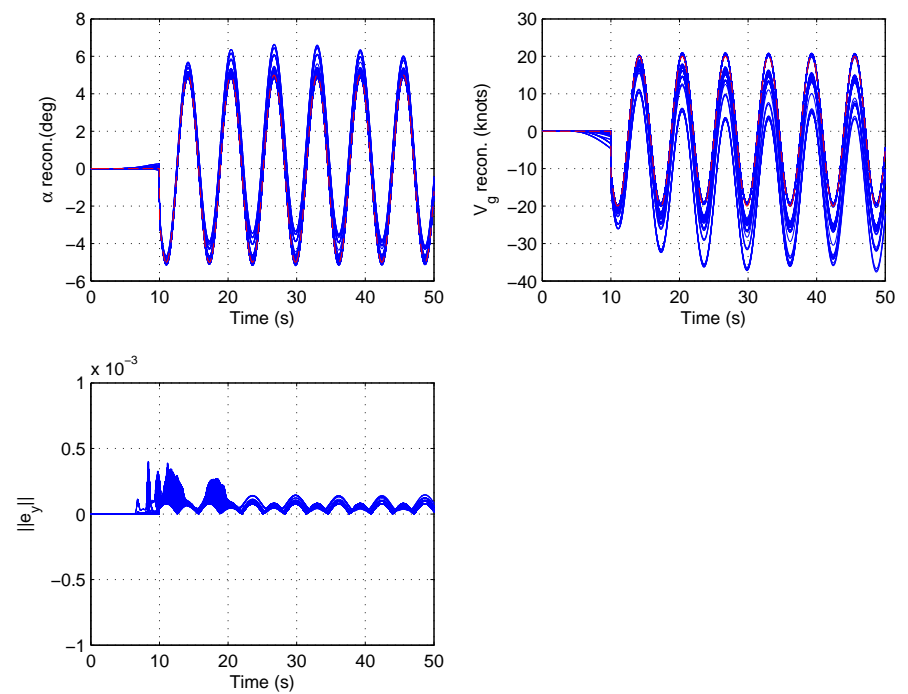

(b) Fault reconstruction (actual (dotted lines) and reconstructed (solid lines) faults) and norm of output error
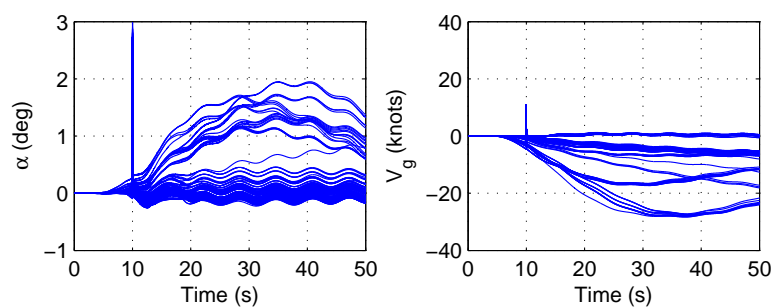

(c) Estimation errors

Fig. 8. Dynamic manoeuvres: oscillatory faults with imperfect scheduling parameters 

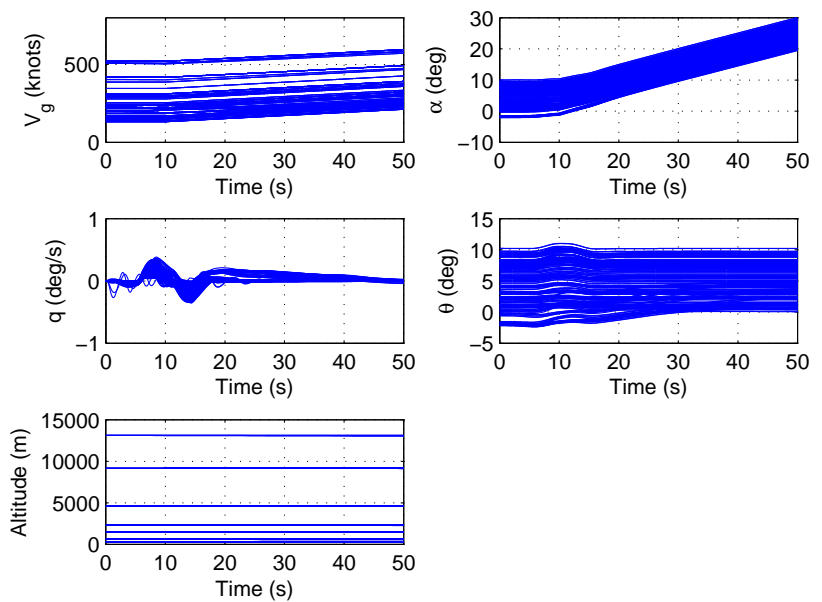

(a) Observer states and altitude
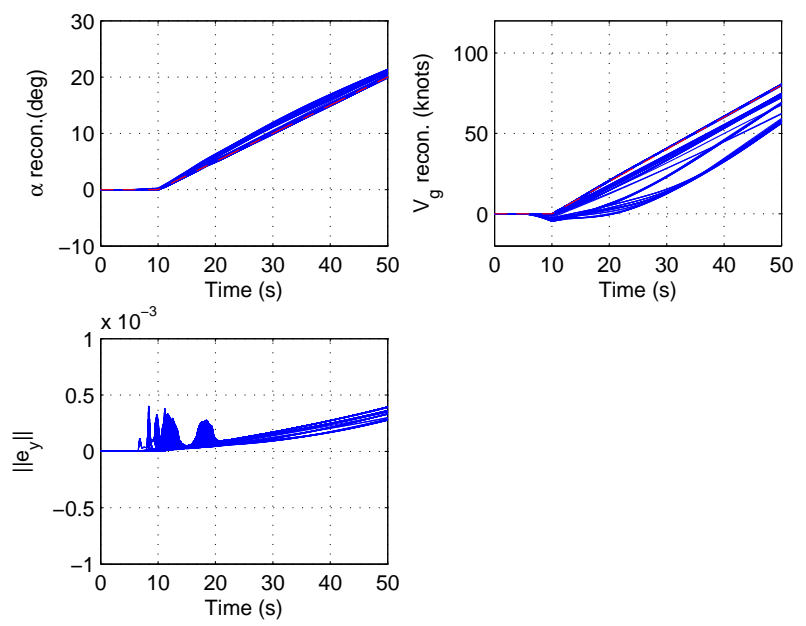

(b) Fault reconstruction (actual (dotted lines) and reconstructed (solid lines) faults) and norm of output error
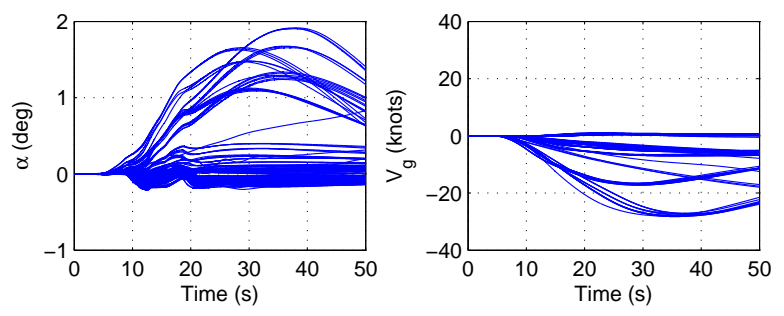

(c) Estimation errors

Fig. 9. Dynamic manoeuvres: drift faults with imperfect scheduling parameters 
[24] M. Sato, Gain-scheduled $H_{\infty}$ filters using inexactly measured scheduling parameters, in: Proceedings of American Control Conference (ACC), 2010, pp. 3088-3093.

[25] M. Sato, D. Peaucelle, Gain-scheduled output-feedback controllers using inexact scheduling parameters for continuous-time LPV systems, Automatica 49 (4) (2013) 1019-1025.

[26] M. J. Lacerda, E. S. Tognetti, R. C. Oliveira, P. L. Peres, $H_{\infty}$ LPV filtering for discrete-time linear systems subject to additive and multiplicative uncertainties in the measurement, in: Proceedings of European Control Conference (ECC), 2013, pp. 1823-1828.

[27] G. Puyou, P. Ezerzere, Tolerance of aircraft longitudinal control to the loss of scheduling information: toward a performance oriented approach, in: 7th IFAC Symposium on Robust Control Design, 2012, pp. 393-399.

[28] P. Antoinette, G. Ferreres, Robust gain scheduling techniques for adaptive control, in: Proceedings of European Control Conference (ECC), 2009.

[29] A. Marcos, S. Ganguli, G. J. Balas, An application of $H_{\infty}$ fault detection and isolation to a transport aircraft, Control Engineering Practice 13 (1) (2005) 105-119.

[30] F.-R. López-Estrada, J.-C. Ponsart, C.-M. Astorga-Zaragoza, J.-L. Camas-Anzueto, D. Theilliol, Robust sensor fault estimation for descriptor-LPV systems with unmeasurable gain scheduling functions: Application to an anaerobic bioreactor, International Journal of Applied Mathematics and Computer Science 25 (2) (2015) 233-244.

[31] K. P. B. Chandra, H. Alwi, C. Edwards, Sliding mode observers for fault detection of uncertain LPV systems with imperfect scheduling parameter knowledge, in: 53rd Annual Conference on Decision and Control (CDC), IEEE, 2014, pp. 4777-4782.

[32] P. Goupil, J. Boada Bauxell, A. Marcos, P. A. N. Rosa, M. L. Kerr, L. Dalbies, An overview of the FP7 reconfigure project: Industrial, scientific and technological objectives, in: Proceedings of 9th IFAC Symposium on Fault Detection, Supervision and Safety of Technical Processes, 2015.

[33] H. Alwi, C. Edwards, Robust actuator fault reconstruction for LPV systems using sliding mode observers, in: 49th IEEE Conference on Decision and Control (CDC), 2010, pp. 84-89.

[34] P. Goupil, J. Boada-Bauxell, A. Marcos, E. Cortet, M. Kerr, H. Costa, Airbus efforts towards advanced real-time fault diagnosis and fault tolerant control, IFAC Proceedings Volumes 47 (3) (2014) 3471-3476.

[35] H. Alwi, L. Chen, C. Edwards, Reconstruction of simultaneous actuator and sensor faults for the reconfigure benchmark using a sliding mode observer, in: 19th IFAC World Congress, 2014.

[36] V. Fernandez, J. Montano, C. Recupero, M. L. Kerr, P. A. N. Rosa, J. Boada Bauxell, L. Dalbies, A tool for industrial verification and benchmarking of FDD/FTC designs, in: Proceedings of 9th IFAC Symposium on Fault Detection, Supervision and Safety of Technical Processes, 2015. 